\title{
3 Research Soure

\section{Variation of soil microbiome in licorice rhizosphere driven with inoculating dark septate endophytes under drought stress}

Chao He ( $\sim$ hc891215@126.com )

Chinese Academy of Medical Sciences \& Peking Union Medical College Institute of Medicinal Plant

Development https://orcid.org/0000-0003-2019-2938

\section{Wenquan Wang}

Chinese Academy of Medical Sciences and Peking Union Medical College

Junling Hou

Beijing University of Chinese Medicine

Xianen Li

Chinese Academy of Medical Sciences and Peking Union Medical College

\section{Research}

Keywords: dark septate endophytes, drought microbiome, Illumina MiSeq sequencing, 16S, ITS2, licorice rhizosphere

Posted Date: October 9th, 2020

DOl: https://doi.org/10.21203/rs.3.rs-87436/v1

License: (1) This work is licensed under a Creative Commons Attribution 4.0 International License. Read Full License 


\section{Abstract}

\section{Background}

Dark septate endophytes (DSE) are facultative biotrophic ascomycetes that colonize plant roots either alone or with arbuscular mycorrhizal (AM) fungi. DSE may provide nutrients to their plant hosts and help them adapt to various abiotic and biotic stresses. DSE inoculation under drought stress increased the biomass, root exudates, and AM fungi in the licorice (Glycyrrhiza uralensis Fisch.) rhizosphere. We conducted a pot experiment to establish whether the responses of licorice to DSE inoculation under drought stress are caused by changes in the rhizosphere microbiome. Each pot was inoculated with either Acrocalymma vagum or Paraboeremia putaminum. One set of pots was inoculated with a sterile culture medium. All three DSE-treated and uninoculated pots were subjected either to a well-watered $(70 \%$ field water capacity, FWC) or drought stress ( $30 \%$ FWC) water regime. Rhizosphere microbiome compositions were measured by Illumina MiSeq sequencing of the 16S and ITS2 rRNA genes.

\section{Results}

In total, 1,278 fungal and 1,583 bacterial operational taxonomic units (OUTs) were obtained at a $97 \%$ sequence similarity level. Ascomycota were the predominant fungi and Proteobacteria, Actinobacteria, Chloroflexi and Firmicutes were the predominant bacteria. DSE inoculation and water regime significantly influenced the rhizosphere microbiome composition. However, the effects of DSE on the fungal community were greater than those on the bacterial community. Paraboeremia putaminum exerted a stronger impact on the licorice rhizosphere microbiome than Acrocalymma vagum under drought stress. The observed changes in edaphic factors (water condition, soil organic matter, available N, available $\mathrm{P}$, and available K) caused by DSE inoculation could be explained by the variations in rhizosphere microbiome composition. A network analysis indicated that DSE inoculation augmented the relative abundance of beneficial symbiotrophic fungi and growth-promoting bacteria but diminished the relative abundance of pathogens in the licorice rhizosphere.

\section{Conclusions}

The present study showed that the licorice rhizosphere microbial community differed between the DSEinoculated and uninoculated plants. DSE had a stronger influence on the fungal than on the bacterial rhizosphere community under drought stress. These give us the guidance to develop biofertilizers with DSE consortia to enhance the cultivation of medicinal plants by shaping soil microbial community structure in dryland agriculture.

\section{Background}

Drought is a major abiotic stressor that limits plant growth and agricultural production in arid and semiarid regions worldwide [1, 2]. Water deficit inhibits root growth and development and decreases plant nutrient and water uptake from the soil [3]. Under drought stress, plants modify their root exudate 
abundance and composition [4]. This response alters the rhizosphere microbiome, which, in turn, strongly influences plant survival and growth and soil ecology [5]. Several studies have shown that plant adaptation to abiotic stress is closely associated with rhizosphere microorganisms [6, 7]. Beneficial endophytic fungal colonization can alter root growth, increase plant biomass, reduce water loss, and help host plants adapt to arid environments $[8,9]$. Moreover, dark septate endophytes (DSE) enhance viral resistance and heavy metal tolerance in plants $[10,11]$.

DSE are ubiquitous root-colonizing fungi characterized by dark, septate hyphae and melanized microsclerotia. They are integral and functional parts of plant roots [12]. DSE inoculation facilitates plant growth, nutrient uptake, and biotic and abiotic stress tolerance $[8,11]$. Previous studies demonstrated that rhizosphere-associated microbes indirectly affect plant growth by changing the rhizosphere microbial community composition and activity. These communities play key roles in soil nutrient cycling and structural formation $[13,14]$. Fiorentino et al. [15] found that Trichoderma inoculation more strongly affected the eukaryotic community composition of low- $\mathrm{N}$ than $\mathrm{N}$-fertilized soils. Changey et al. [16] found that arbuscular mycorrhizal (AM) fungal inoculation dramatically influenced the rhizosphere microbial community composition. Tian et al. [17] reported that Glomus intraradices inoculation increased certain beneficial bacterial species and decreased certain pathogenic fungi in the Panax ginseng rhizosphere. Han et al. [18] proposed that Bacillus amyloliquefaciens promotes cucumber growth by modulating its rhizosphere microbial community composition. The direct effects of beneficial rhizosphere microbes on plant growth have been extensively examined $[19,20]$. Nevertheless, there is relatively little information on the impact of DSE inoculation on the rhizosphere microbial community and its network structure.

Licorice (Glycyrrhiza uralensis Fisch.) belongs to Fabaceae. It is widely distributed in arid and semi-arid regions worldwide. It is regarded as an "essential herbal medicine" in traditional Chinese medicine and has been prescribed and administered for $>1,000 \mathrm{y}$. Its active principles include glycyrrhizin and glycyrrhizic acid [21]. Licorice adapts very well to low-fertility soils and drought. In fact, it has been used in the ecological restoration of arid regions [22]. In its natural habitats, the licorice rhizosphere harbors actinobacteria, rhizobia, other soil bacteria, AM, and DSE with plant growth-promoting activity [23, 24]. An ester-linked phospholipid fatty acid (PLFA) analysis in our previous study showed that DSE (Acrocalymma vagum and Paraboeremia putaminum) inoculation increased AM and gram-negative bacterial abundance in the licorice rhizosphere under drought stress [8]. However, its effects on the composition of the microbial communities in the licorice rhizosphere remain unclear. Rhizosphere microbes play vital roles in plant growth, health, and abiotic stress tolerance. Here, we used Illumina MiSeq sequencing to reveal variations in the composition of the licorice rhizosphere microbial communities inoculated with DSE under drought stress. We hypothesized that DSE inoculation considerably alters the composition and network structure of the fungal and bacterial communities in the licorice plant rhizosphere. Furthermore, these changes are more pronounced under drought stress than well-watered conditions. Our objective was to test whether DSE inoculation alters the soil microbiome and increases drought tolerance in licorice. 


\section{Results}

\section{Characterization of Illumina sequencing data}

We obtained 2,304,715 fungal and 2,098,398 bacterial sequences. Rarefaction curve analysis displayed high 16S rRNA gene sequencing depth and strong potential for observing community diversity in each licorice rhizosphere (Fig. 1). Rank abundance curves showed that all six treatments had high species evenness and homogeneity (Fig. 2). The sequencing results covered the biological information of most of the fungi and bacteria in the soil samples.

After filtering 1,266,009 low-quality sequences, 1,568,052 effective fungal and 987,120 effective bacterial sequences were clustered into 1,278 fungal and 1,583 bacterial operational taxonomic units (OTUs) at $97 \%$ sequence similarity. Of the 1,278 fungal OTUs, 196 occurred in all six treatments while 85, 94, 48, 21, 202, and 33 OTUs were found only in the uninoculated condition (NCK), P. putaminum inoculation (NPP), and $A$. vagum inoculation (NAV) under well-watered and in the three DSE inoculation treatments DCK, DPP, and DAV under drought stress, respectively (Fig. 3). Of the 1,583 bacterial OTUs, 1,082 were detected in all treatments and $31,45,71,36,64$, and 38 existed only in NCK, NPP, NAV, DCK, DPP, and DAV, respectively (Fig. 3).

\section{Rhizosphere microbial diversity and richness}

Under well-watered, inoculation with either P. putaminum or $A$. vagum increased the soil Simpson fungus index compared to the uninoculated condition. The $A$. vagum inoculation decreased the soil fungus Ace and Chao1 indices. Compared with well-watered, drought stress decreased the diversity and richness of the fungal community colonizing the licorice rhizosphere. Compared to the uninoculated condition, $P$. putaminum inoculation increased the soil fungus Shannon, Ace, and Chao1 indices but decreased the soil fungus Simpson index. However, $A$. vagum inoculation increased the soil fungus Ace and Chao1 indices (Table 1).

DSE inoculation significant affected soil bacterial diversity and richness under all water regimes (Table 2). Under well-watered, DSE inoculation increased the soil bacteria Chao1 index but decreased the soil bacteria Simpson index compared with the uninoculated condition. Under drought stress, P. putaminum inoculation increased the soil bacteria Ace and Chao1 indices whereas $A$. vagum inoculation had no significant effect on soil bacterial community diversity and richness. Compared with the uninoculated condition, drought stress decreased the soil bacteria Simpson index.

\section{Rhizosphere microbial community composition}

A total of 1,278 fungal OTUs were found in the licorice rhizosphere and classified as Ascomycota, Basidiomycota, Zygomycota, Glomeromycota, and certain unknown fungi. Ascomycota and Basidiomycota were identified in all treatments. Ascomycota was the dominant fungal phylum and its relative abundance range was $89.9-99.6 \%$ across the various treatments (Fig. 4). A heatmap based on the top 50 abundant fungal genera revealed that the colonization of certain relatively abundant fungi 
varied among treatments (Fig. 5). Of the 50 abundant fungal genera, five OTUs (Aspergillus OTU86, Fusarium 0TU1748, Gibberella 0TU1913, Trichoderma 0TU8380 and unclassified_f_Chaetomiacease OTU936) were distributed mainly in NCK, four OTUs ( Acremonium OTU454, Chaetomium OTU1508, Phialophora OTU1296 and Stachybotrys OTU1633) were distributed mainly in NPP, two OTUs (Aspergillus OTU86 and Trichoderma OTU8380) were distributed mainly in NAV, three OTUs (Aspergillus OTU86, Trichoderma OTU8380 and unclassified_f_Microascaceae OTU1963) were distributed mainly in DCK, four OTUs (Chaetomium OTU1508, Pseudalescheria OTU946, unclassified_f_Chaetomiaceae OTU936 and unclassified_k_Fungi OTU1449) were distributed mainly in DPP, and two OTUs (Aspergillus OTU86 and Trichoderma OTU8380) were distributed mainly in DAV (Fig. 5).

A total of 1,583 bacterial OTUs were detected in the licorice rhizosphere. The dominant bacterial phyla were Proteobacteria, Actinobacteria, Chloroflexi, Firmicutes, Acidobacteria, Cyanobacteria, Bacteroidetes, Gemmatimonadetes, Planctomycetes, Verrucomicrobia, Nitrospirae, Saccharibacteria, and certain unknown bacteria. The relative abundance of these bacterial phyla varied among treatments (Fig. 4). Of the 50 dominant bacterial genera, nine OTUs (Bacillus OTU4110, Devosia OTU1666, Nocardioides OUT5635, Oscillatoria OTU6539, Pseudarthrobacteria OTU3533, Sphingomonas OTU5209, norank-cAcidobacteria OTU1690, norank-c-Gitt-GS-136 OTU3951 and norank-o-JG30-KF-CM450TU6085) were distributed mainly in NCK, six OTUs (Bacillus OTU4110, Pseudarthrobacteria OTU3533, Pseudomonas OTU5762, norank-c-Acidobacteria OTU1690, norank-f- Anaerolineaceae OTU1186 and norank-c-KD4-96 OTU3568) were distributed mainly in NPP, eight OTUs (Bacillus OTU4110, Devosia OTU1666, Ensifer OTU6341, Paenibacillus OTU4751, norank-o-Acidmicrobiales OTU4282, norank-c- Acidobacteria OTU1690, norank-f-Anaerolineaceae OTU1186 and norank-o-JG30-KF-CM45 OTU6085) were distributed mainly in NAV, seven OTUs (Bacillus OTU4110, Nocardioides OTU5635, Paenibacillus OTU4751, norank-cAcidobacteria 1690, norank-o-Acidmicrobiales OTU4282, norank-c-Gitt-GS-136 OTU3951 and norank-cKD4-96 OTU3568) were distributed mainly in DCK, six OTUs (Bacillus OTU4110, Lamia OTU4301, Pseudomonas OTU5762, norank-c-Acidobacteria OTU1690, norank-f-Anaerolineaceae OTU1186 and norank-c-KD4-96 OTU3568) were distributed mainly in DPP, and 11 OTUs (Bacillus OTU4110, Lysinibacillus OTU9205, Microbacterium 0TU1007, Nocardioides OTU5635, Paenibacillus OTU4751, Sporocytophaga OTU2217, norank-c-Acidobacteria OTU1690, norank-o-Acidmicrobiales OTU4282, norank-f-Anaerolineaceae OTU1186, norank-c-KD4-96 OTU3568 and norank-c-Gitt-GS-136 OTU3951) were distributed mainly in DAV (Fig.5).

Nonmetric multidimensional scaling (NMDS) ordination revealed that the rhizosphere fungal community composition significantly differed between the DSE inoculation and uninoculated treatments under wellwatered (Fig. 6). Compared to the uninoculated condition, P. putaminum inoculation substantially affected fungal community composition under drought stress. No dramatic effect was observed in response to $A$. vagum inoculation (Fig. 6). DSE inoculation significantly affected the composition of the bacterial community under well-watered. The $A$. vagum and $P$. putaminum inoculation had different effects (Fig. 6). Under drought stress, P. putaminum inoculation significantly affected the bacterial community composition relative to the uninoculated condition. In contrast, $A$. vagum inoculation had no significant impact on the bacterial community composition (Fig. 6). Permutational multivariate analysis 
of variance (PerMANOVA) indicated that the fungal $\left(F=7.435, R^{2}=0.264, P=0.001 ; F=6.224, R^{2}=0.331\right.$, $P=0.001)$ and bacterial $\left(F=6.125, R^{2}=0.392, P=0.001 ; F=5.648, R^{2}=0.440, P=0.001\right)$ community compositions were significantly different between the well-watered and the drought stress treatments. Furthermore, drought stress and DSE inoculation more strongly affected the fungal than the bacterial community composition.

\section{Edaphic factor and rhizosphere microbe preferences}

An edaphic factor/microbe preference analysis showed that the fungi and bacteria colonizing the licorice rhizosphere significantly preferred the soil moisture, organic matter content, and available N, P, and K (Fig. 7). Of the 50 relatively abundant fungal OTUs, two (Gibberella OTU1913 and Phialophora OTU1296) had a significant positive preference for soil moisture but OTU575 (Vermispora) presented with a significant negative moisture preference. 0TU308 (Guehomyces) showed negative available N preference. Three OTUs (Guehomyces OTU308, unclassified_f_Lasiosphaeriaceae OTU161, and Schizothecium 0TU474) showed negative available P preferences. OTU1389 (Humicola) displayed a positive available K preference while OTU1539 (Mycoarthris) displayed a positive soil organic matter preference (Fig. 7). Of the 50 relatively abundant bacterial OTUs, OTU3533 (Pseudarthrobacter) and three OTUs (Actinobacteria OTU1174, Streptomyces 0TU5992 and Xanthomonadales 0TU7347) showed positive and negative moisture preferences, respectively. Two OTUs (norank-f-Anaerolineaceae OTU1186 and Pseudomonas OTU5762) positively correlated with available N. Seven OTUs (Acidimicrobiales OTU4282, Gaiella OTU 8721, lamia OTU 4301, Oscillatoria OTU6539, norank-f-Gemmatimonadaceae OTU1174, norank-fNitrosomonadaceae 0TU732 and Streptomyces OTU5992) had a significant negative correlation with available K. Three OTUs (norank-c-Cynobacteria OTU5893, norank-f-Anaerolineaceae OTU1186 and norank-f-Sandaracinaceae OTU476) and one OTU (Lysinibacillus OTU9205) showed positive and negative organic matter preferences, respectively (Fig.7).

\section{Structure of various treatment-rhizosphere microbe networks}

The various treatment-rhizosphere fungal networks are shown in Fig. 8. Under well-watered, P. putaminum inoculation increased the relative symbiotroph abundance but decreased the relative abundance of saprotroph and other fungi. In contrast, $A$. vagum inoculation increased the relative abundance of symbiotrophs and other fungi and decreased the relative abundance of pathotrophs and saprotrophs compared with the uninoculated condition. Drought stress decreased the relative abundance of symbiotrophs, saprotrophs, and other fungi but increased the relative abundance of pathotrophs compared with the well-watered condition. However, P. putaminum inoculation increased the relative abundance of symbiotrophs and other fungi and decreased the relative abundance of pathotrophs. Moreover, $A$. vagum inoculation increased the relative abundance of symbiotrophs, saprotrophs, and other fungi and decreased the relative abundance of pathotrophs compared with the uninoculated condition. 
The network of different treatments-rhizosphere bacteria is shown in Fig. 8. Bacillus,Pseudarthrobacter OTU3533, and other bacteria had variable relative abundance under different treatments. Oscillatoria was only distributed under NCK. Microbacterium OUT1007 was distributed under NCK, NAV, DCK, and DAV. Inoculation with $A$. vagum decreased the relative abundance of Oscillatoria compared with the uninoculated condition. Nocardioides was only distributed under DCK and DAV. Sporocytophaga was only detected under DAV. Arcnimonas only occurred under NAV and DAV. Ensifer was found under NPP, NAV, and DPP. Pedomicrobium was only seen under NPP.

The network of various treatment-rhizosphere microbe functional group is shown in Fig. 8. Compared to the uninoculated treatment, under well-watered, DSE inoculation increased the relative abundance of beneficial and neutral fungi and bacteria and decreased the relative abundance of pathogenic fungi and bacteria. Relative to the uninoculated condition, under drought stress, $P$. putaminum inoculation decreased the relative abundance of pathogenic fungi and beneficial bacteria but increased the relative abundance of neutral fungi and neutral and pathogenic bacteria. The A. vagum inoculation increased the relative abundance of beneficial fungi and neutral bacteria but decreased the relative abundance of pathogenic fungi and bacteria.

\section{Discussion}

The plant rhizosphere houses a complex microbiome that includes bacteria, archaea, and fungi. These microorganisms affect plant survival, growth, and adaptability $[25,26]$. Here, we used Illumina MiSeq sequencing to reveal the changes in microbial community diversity and network structure in the rhizosphere of licorice inoculated with DSE under drought stress. The relative abundance of microbes colonizing licorice rhizosphere differed among treatments. However, Ascomycota, Proteobacteria, Actinobacteria, Chloroflexi, and Firmicutes were the dominant phyla under both well-watered and drought conditions. The predominance of Ascomycota in arid and semi-arid regions was previously reported [27, 28]. Dai et al. [29] found that Actinobacteria, Proteobacteria, Saccharibacteria, Chloroflexi, Acidobacteria, and Cyanobacteria were the predominant phyla in drought-treated and untreated peanut rhizosphere. Lundberg et al. [30] observed that Proteobacteria, Bacteroidetes, Actinobacteria, Acidobacteria, Firmicutes, Gemmatimonadetes, and Cyanobacteria dominated in Arabidopsis rhizosphere. Barraza et al. [31] also reported that the bacterial community structure of the common bean roots, mainly composed by Proteobacteria, Actinobacteria, Bacteroidetes, Acidobacteria, and Firmicutes. The major rhizosphere microbes vary widely among plant species. Nevertheless, Actinobacteria and Proteobacteria may be the most common bacterial phyla in plant rhizospheres.

Biotic and abiotic stressors alter rhizosphere microbe community structure and may augment or diminish certain microbial populations [32,33]. He et al. [8] found that interactions between DSE inoculation and water regime markedly influenced the soil organic matter content. Compared to the uninoculated condition, $A$. vagum inoculation under drought conditions increased soil available $\mathrm{N}$ and $\mathrm{P}$. In contrast, $P$. putaminum inoculation decreased soil organic matter and available $\mathrm{N}$ relative to the uninoculated condition. The microbes colonizing the licorice rhizosphere exhibited distinct preferences for various soil 
factors. For instance, Phialophora OTU1296, Gibberella OTU1913, and Pseudarthrobacter OTU3533 showed a positive moisture preference whereas Vermispora OTU575, Xanthomonadales OTU7347, Actinobacteria OTU1174, and Streptomyces OTU5992 displayed a negative moisture preference. The norank-f-Anaerolineaceae OTU1186, norank-f-Sandaracinaceae OUT476, Mycoarthris OUT1539, and norank-c-Cynobacteria OTU5893 showed a positive soil organic matter preference while Lysinibacillus OTU9205 presented with a negative soil organic matter preference. These findings were consistent with those previously reported that microbial inoculation broadly influences plant rhizosphere microbial communities by altering soil chemical properties and indirectly affecting host plant growth $[34,35]$.

Here, NMDS ordination disclosed that $P$. putaminum inoculation had a significant effect on fungal community composition under drought stress. However, no significant difference was found between $A$. vagum inoculation and the uninoculated condition even though $A$. vagum inoculation increased the fungal community Ace and Chao1 indices in the licorice rhizosphere. The P. putaminum inoculation increased the relative abundance of Basidiomycota and Zygomycota but decreased the relative abundance of Ascomycota in the licorice rhizosphere under drought stress. Previous studies showed that the three aforementioned fungal phyla predominated in different ecological environments [36]. Certain Ascomycota including DSE form mycorrhizae in plant roots and enhance plant nutrient uptake and growth [37, 38]. Lin et al. [39] found that Ascomycota, Basidiomycota, and Zygomycota strongly tolerated heavy metal contamination. Moreover, Proteobacteria, Actinobacteria, Chloroflexi, Firmicutes, and Acidobacteria predominated in the licorice rhizosphere under drought stress regardless of DSE inoculation. Compared to the uninoculated condition, inoculation with $P$. putaminum increased the relative abundance of Acidobacteria, Chloroflexi, and Cyanobacteria but decreased the relative abundance of Actinobacteria. The $A$. vagum inoculation increased the relative abundance of Cyanobacteria and Bacteroidetes. Khan et al. [40] reported that Acidobacteria, Actinobacteria, Bacteroidetes, and Proteobacteria were highly abundant in medicinal plant rhizosphere microbiomes in arid soil. Proteobacteria, Acidobacteria, and Bacteroidetes had high heavy metal tolerance [41]. Sanguin et al. [42] found that Proteobacteria enrichment increased the disease suppression capacity of the rhizosphere. Singh [43] reported that Cyanobacteria improved the soil environment and survived in arid soil by accumulating soil carbon and nitrogen. The P. putaminum inoculation increased Chloroflexi abundance which was consistent with a previous study on microflora in a biofertilizer soil [44]. Wu et al. [45] found that Chloroflexi does not produce oxygen during photosynthesis and inhibits nitrogen fixation. In contrast, Acidobacteria decompose cellulose and modulate soil pH. Hence, the enrichment of specific rhizosphere fungi and bacteria might enable plants to maintain active microbiomes that improve survival under drought stress [46].

It was unknown whether DSE-mediated changes in the rhizosphere microbial communities augment drought stress tolerance in licorice. Our previous study showed that DSE inoculation ( $A$. vagum and $P$. putaminum) improve licorice growth and survival under drought conditions [8]. In the present study, a network structure analysis disclosed that both soil fungal and bacterial networks were characterized by high specialization and modularity. Drought stress markedly affected the relative abundance of various microbial functional groups. Compared to the uninoculated condition under drought stress, P. putaminum 
and $A$. vagum inoculation increased symbiotrophic fungi by $78.2 \%$ and $34.6 \%$ and saprotrophic fungi by $0.4 \%$ and $12.1 \%$ and decreased pathotrophic fungi by $125.6 \%$ and $44.5 \%$, respectively. Microascaceae, Trichoderma, and Aspergillus were the dominant fungi in DCK, Chaetomium and Pseudalescheria predominated in DPP, and Trichoderma and Aspergillus prevailed in DAV. The Microascaceae include both saprobes and plant pathogens. Certain species are intrinsically resistant to antifungal agents $[47,48]$. Trichoderma is an effective biofertilizer, soil amendment, and biocontrol agent [49]. Certain Aspergillus species such as $A$. flavus are facultative plant pathogens under drought stress and can produce considerable amounts of aflatoxin [50]. In contrast, A. niger and A. fumigatus are metallotolerant [41]. Chaetomiaceae degrade cellulose in the soil and increase soil organic matter [51]. Pseudallescheria (Scedosporium) spp. are global pathogens that resistant most antifungal agents [52].

Compared to the fungal community, DSE inoculation and the water regime had less influence on the composition of the bacterial community colonizing the licorice rhizosphere. Bacillus, Microbacterium, Nocardioides, and Pseudarthrobacter predominated and constituted $78.6 \%$ of the total abundance under the DCK treatment. Bacillus, Pseudarthrobacter, and Ensifer occupied $54.6 \%$ of the total abundance under the DPP treatment. Bacillus, Microbacterium, Nocardioides, Pseudarthrobacter, Sporocytophaga, and Arenimonas comprised $81.3 \%$ of the total abundance under the DAV treatment. These bacteria have high drought stress tolerance and most of them promote plant growth [53-55]. Bacillus species are also effective biocontrol bacteria [56]. Pseudarthrobacter and Sporocytophaga efficiently degrade cellulose, crude oil, and multibenzene compounds $[57,58]$. Compared to the uninoculated condition, P. putaminum and $A$. vagum inoculation increased the relative abundance of beneficial ( $2.5 \%$ and $56.3 \%)$ and neutral ( $86.4 \%$ and $4.1 \%$ ) fungi but decreased the relative abundance of pathogenic fungi (178.5\% and $50.7 \%$ ) under drought stress. Compared to the uninoculated condition, $P$. putaminum inoculation increased the relative abundance $(107.4 \%$ and $55.4 \%)$ of neutral and pathogenic bacteria but decreased the relative abundance $(92.8 \%)$ of beneficial bacteria under drought stress. The $A$. vagum inoculation increased the relative abundance $(45.8 \%)$ of neutral bacteria but decreased the relative abundance $(128.6 \%)$ of pathogenic bacteria. Our results indicated that DSE inoculation alters the licorice rhizosphere microbiome community composition. It enriched beneficial and neutral fungi and reduced harmful fungi under drought stress. Hence, DSE inoculation may be an important modality for the improvement of plant growth and drought resistance $[8,59,60]$.

\section{Conclusion}

DSE inoculation and water regime markedly affected the composition and diversity of the microbial communities colonizing licorice rhizospheres, and such impact on fungal community was greater than bacterial community. Of the two DSE species, P. putaminum exerted a stronger influence on the licorice rhizosphere microbiome than $A$. vagum under drought stress. The edaphic factor changes caused by DSE inoculation and water regime partially account for the observed variations in licorice rhizosphere microbiome. DSE inoculation under drought stress enriched beneficial symbiotrophic fungi and growthpromoting bacteria but decreased the relative abundance of licorice rhizosphere pathogens. In this manner, it promoted licorice growth, strengthened pathogen resistance and drought tolerance, and 
facilitate licorice survival under drought stress. These guide us to develop efficient and ecofriendly biofertilizers with symbiotic fungal consortia for the cultivation of medicinal plants based on the soil characteristics and the microbial community that it harbors in dryland agriculture.

\section{Methods}

\section{Biological materials and growth substrates}

The DSE strains, Acrocalymma vagum and Paraboeremia putaminum, were isolated from licorice roots naturally growing on the arid arable land of Northern China. They were deposited in the Laboratory of Endangered Species Breeding Engineering of the Institute of Medicinal Plant Development of the Chinese Academy of Medical Sciences and Peking Union Medical College, Beijing, China [8]. Licorice seeds were provided by China National Traditional Chinese Medicine Corporation and stored at $4{ }^{\circ} \mathrm{C}$.

The substrate used for the pot experiment was a 1:2 (w/w) mixture of sand $(<2 \mathrm{~mm})$ and soil collected from the arid arable land of Northern China whereupon the licorice plants were naturally distributed. The physicochemical properties of the substrate were organic matter content, $21.57 \mathrm{mg} \mathrm{g}^{-1}$; available nitrogen (N), $85.19 \mathrm{mg} \mathrm{kg}^{-1}$; and available phosphorus (P), $7.90 \mathrm{mg} \mathrm{kg}^{-1}$.

\section{Experimental setup}

The experiment had a randomized, complete factorial design with two factors: (1) DSE inoculation (including Acrocalymma vagum (AV) inoculation, Paraboeremia putaminum (PP) inoculation, and uninoculated control (CK)) and (2) water regime (including well-watered (WW) and drought stress (DS)). Each of the six treatments had five replicates and there were 30 pots in total [8].

Two licorice seedlings were transplanted to a sterile plastic pot $(13 \mathrm{~cm}$ diameter $\times 12 \mathrm{~cm}$ height) filled with $800 \mathrm{~g}$ of nonsterile growth substrate. For the DSE inoculation treatments, $600 \mathrm{~g}$ of the growth substrate was poured into a pot, and 5-mm plugs were excised from the edges of actively growing DSE colonies on the culture media. The plugs were inoculated at 1-cm intervals near the licorice seedling roots. Then, $200 \mathrm{~g}$ of the growth substrate was added. For the uninoculated control, two plugs were excised from the fungus-free sterile medium and inoculated near the licorice seedling roots in each pot. The entire inoculation process was performed on a clean bench. All pots were maintained in a growth chamber under a $14 \mathrm{~h} / 10 \mathrm{~h}$ photoperiod, $27^{\circ} \mathrm{C} / 22{ }^{\circ} \mathrm{C}$ (day/night), and $60 \%$ mean $\mathrm{RH}$.

After $1 \mathrm{mo}$, half the DSE-inoculated and uninoculated pots were subjected to the WW treatment ( $70 \%$ field water capacity), while the balance were subjected to the DS treatment (30\% field water capacity). Drought stress was applied according to the median soil moisture content recorded for the natural licorice habitat in Northern China. The soil moisture content in each pot was measured using a soil humidity recorder (L99-TWS-2; Hangzhou Loggertech Co. Ltd., Hangzhou, China). Lost water was replenished with sterile distilled water to maintain the desired field capacity determined by regular weighing. Seedlings were grown for 3 mo. 


\section{Rhizosphere soil sampling and physicochemical properties}

The rhizosphere soil strongly adhering to the root surfaces was collected from each pot. Each soil sample was passed through a 2-mm sieve and divided into two subsamples. One was naturally dried at about 25 ${ }^{\circ} \mathrm{C}$ and its physicochemical properties were measured. The other was frozen at $-80{ }^{\circ} \mathrm{C}$ until the subsequent microbial community composition analysis. A 0.2-g dried soil sample was digested in $10 \mathrm{~mL}$ of a 10:1:2 mixture of perchloric acid (12.7 M), sulfuric acid (18 M), and water in a Mars 6 microwave reaction system (CEM Corporation, Matthews, NC, USA) until a clear liquid was obtained. The soil organic matter content, available nitrogen $(\mathrm{N})$, available phosphorus $(\mathrm{P})$, and available potassium $(\mathrm{K})$ were quantified by dichromate oxidization in the presence of sulfuric acid [61], alkaline hydrolysis diffusion, chlorostannous-reduced molybdophosphoric blue [62], and flame photometry [63], respectively.

\section{Molecular analysis}

The total genomic DNA from 0.25-g soil samples was extracted with a Powersoil ${ }^{\circledR}$ DNA Extraction kit (Mo Bio, Carlsbad, CA, USA). The DNA quality was tested by $0.1 \%(\mathrm{w} / \mathrm{v})$ agarose gel electrophoresis. The DNA purity and concentration were measured with a NanoDrop ${ }^{\mathrm{TM}} 1000$ spectrophotometer (Thermo Fisher Scientific, Waltham, MA, USA). Two universal primers 338F (5'-ACTCCTACGGGAGCAG CAG-3')-806R (5'GGACTACHGGGTWTCTAAT-3') [64] and ITS1F (5'-CTTG GTCATTTAGGAAGTAA-3')-ITS2R(5'GCTGCGTTCTTCATCATGATGC-3') [65] characterized the microbial communities by targeting the bacterial 16S rRNA genes $v 3-v 4$ and the fungal ITS1 and ITS2 (internal transcribed spacer) regions, respectively. PCR was conducted in triplicate in a $20-\mu \mathrm{L}$ reaction system containing $4 \mu \mathrm{L}$ of $5 \times$ FastPfu Buffer (for 16s v3-v4) / $2 \mu \mathrm{L}$ of 10x Buffer (for ITS), $2 \mu \mathrm{L}$ dNTPs, $0.8 \mu \mathrm{L}$ of the aforementioned forward and reverse primers, $0.4 \mu \mathrm{L}$ FastPfu Polymerase (16S v3-v4) / $0.2 \mu \mathrm{L}$ rTaq polymerase (ITS), $0.2 \mu \mathrm{L}$ bovine serum albumin (BSA), and $10 \mathrm{ng}$ template DNA. PCR amplification was conducted using the following thermal program: initial denaturation at $95^{\circ} \mathrm{C}$ for $3 \mathrm{~min}$ followed by 28 denaturation cycles at $95^{\circ} \mathrm{C}$ for $30 \mathrm{~s}$, annealing at $55^{\circ} \mathrm{C}$ for $30 \mathrm{~s}$, elongation at $72{ }^{\circ} \mathrm{C}$ for $45 \mathrm{~s}$, and a final extension at $72{ }^{\circ} \mathrm{C}$ for $10 \mathrm{~min}$. The PCR products were detected by gel electrophoresis ( $2 \%(\mathrm{w} / \mathrm{v})$ agarose), purified with an AxyPrep $^{\mathrm{TM}}$ DNA Gel Extraction kit (Axygen BioSciences Inc., Union City, CA, USA), and quantified in a QuantiFluor $^{\text {TM }}$ dsDNA system fitted with a QuantiFluor ${ }^{\text {TM }}$-ST fluorometer and a PCR tube adapter (Promega Corporation, Madison, WI, USA). The sample was sequenced using the paired end option $(2 \times 300 \mathrm{bp})$ of an Illumina MiSeq PE 300 platform (IIlumina, San Diego, CA, USA) at the Environmental Genome Platform of Meiji Biomedical Technology Co. Ltd. (Shanghai, China).

\section{Bioinformatics analysis}

Raw fastq files were demultiplexed, quality-filtered, and merged by Trimmomatic and fast length adjustment of short reads (FLASH; Johns Hopkins University, Baltimore, MD, USA) [66]. Sequences that were $<50$ bp long and had average quality score $<20$ or ambiguous bases were removed. The filtered high-quality sequences were merged according to the overlap sequences between read pairs. Sequences with mismatches along the primer region were removed before the downward process. Non-chimeric 
sequences were dereplicated and singletons were discarded. The filtered non-chimeric sequences were clustered into operational taxonomic units (OTUs) at a $97 \%$ sequence level based on the UPARSE pipeline using USEARCH v. 8.0. The RDP Bayesian classifier algorithm was used to classify OTU representative sequences via the fungal (ITS) UNITE database v. 18.11.2018 and the Silva (SSU123) 16S rRNA reference database at confidence threshold $=0.7$. The RDP then collated the functional gene database from GeneBank (Release 7.3; http://fungene.cme.msu.edu/) and obtained species annotation data. To eliminate potential bias caused by divergent sequence depth across samples, all samples were subsampled to the minimum sequencing depth. The dilution curve, the Venn map, and the community composition analysis were conducted in R ( $\mathrm{R}$ Core Team, Vienna, Austria; version 3.4.0) based on the OTU count and associated taxonomy tables. The Alpha diversity index was calculated in mothur (v. 1.30.2). Other statistical analysis was performed in SPSS v. 22.0 (IBM Corp., Armonk, NY, USA) and the remaining graphs were generated with Origin v. 9.0 (OriginLab, Northampton, MA, USA) [67].

\section{Abundance and diversity analyses}

To characterize microbial diversity, the Chao1, Ace, Shannon, and Simpson indices were calculated based on the OTU data. Chao1 and Ace reflect community abundance while Shannon and Simpson indicate community diversity [68]. Rank abundance and rarefaction curves generated in QIIME estimate species evenness and evaluate species richness and sequence depth, respectively [69]. Fungal or bacterial OTU richness was defined as the number of fungal or bacterial OTUs per sample. The relative abundance of a specific fungal or bacterial OTU and class was defined as the ratio of corresponding sequences and class to the total reads PER sample. Each representative OTU sequence in this study was used for taxonomic identification at the phylum, class, order, family, and genus levels.

\section{Statistical methods}

Two-way analysis of variance (ANOVA) was used to disclose the effects of DSE inoculation, water regime, and their interactions on fungal and bacterial OTU diversity. Data shown in the figures are means of $\geq 3$ replicates. Variations among treatment means were compared using Tukey's honestly significant difference (HSD) tests at $P<0.05$. Non-metric multidimensional scaling (NMDS) was used to visualize compositional dissimilarities in the rhizosphere fungal and bacterial communities. The metaMDS command in the vegan package v. 2.4-1 was used [70]. To evaluate the effects of DSE inoculation on the rhizosphere fungal and bacterial communities, permutational multivariate analysis of variance (PerMANOVA) was run using the adonis command in the vegan package with 999 permutations [70]. Rarefaction curves for the bacterial and fungal OTUs were calculated using the specaccum function in the vegan package [70]. The edaphic factors such as water condition, soil organic matter, available $\mathrm{N}$, available $\mathrm{P}$, and available $\mathrm{K} /$ microbe preference analysis was performed according to Yao et al. [71] and Huang et al. [72].

To visualize the structure of the rhizosphere bacterial and fungal networks among treatments, a network was drawn on the basis of genera with abundance $>200$ for OTU-level matrices. To this end, the Prefuse Force Directed OpenCL Layout in CYTOSCAPE v. 3.4.0 was used [73]. 
To identify the beneficial, harmful, and neutral fungi and bacteria, a network analysis was conducted on the abundance of the genera that significantly differed among treatments $[74,75]$. The online FUNGuild application (http://www. stbates.org/ guilds/app.php) was used to assign trophic status to frequent OTUs [74]. The trophic status was assigned with different certainty levels (possible, probable, or highly probable) based on a combination of the aforementioned effects. The community designating the nutrient type as a compound nutrient type was included in "other fungi". The community identified as a compound multifunctional method was unified into "other pathogens/saprophytic fungi" under the nutrient type $[74,75]$.

\section{Abbreviations}

AM, arbuscular mycorrhizae; AV, Acrocalymma vagum; BSA, bovine serum albumin; CK, uninoculated control; DS, drought stress; DSE, dark septate endophyte; OTU, operational taxonomic unit; PLFA, phospholipid fatty acid; PP, Paraboeremia putaminum; WW, well-watered; NCK, non-inoculation under well-watered condition; NPP, inoculation with Paraboeremia putaminum under well-watered condition; NAV, inoculation with Acrocalymma vagum under well-watered condition; DCK, non-inoculation under drought stress; DPP, inoculation with Paraboeremia putaminum under drought stress; DAV, inoculation with Acrocalymma vagum under drought stress.

\section{Declarations}

\section{Ethics approval and consent to participate}

Not applicable.

\section{Consent for publication}

Not applicable.

\section{Availability of data and materials}

The Illumina MiSeq sequence datasets are available at the NCBI Sequence Read Archive BioProject ID PRJNA664875

\section{Competing interests}

The authors declare that they have no competing interests.

\section{Funding}

This research was financially supported by the National Key R \& D Program of China (No. 2017YFC 1700706, 2018YFC1706500).

\section{Authors' contributions}


$\mathrm{CH}$ and WQW designed the experiment; $\mathrm{CH}$ and JLH collected the samples; $\mathrm{CH}$, JLH and XEL performed the laboratory work; and $\mathrm{CH}, \mathrm{WQW}$ and XEL analyzed the data and wrote the manuscript. All authors read and approved the final manuscript.

\section{Acknowledgements}

The authors thank postgraduate students Ying Ren, Min Li, and Xue Zhang of Hebei University for sampling and laboratory work. We thank Dr. Jun Yu of Guizhou Medical University for his valuable suggestions on the organization of this paper. We additionally thank International Science Editing Ltd. for the language editing service.

\section{References}

1. Huang J, Yu H, Guan X, Wang G, Guo R. Accelerated dryland expansion under climate change. Nat Clim Change. 2016;6:166-172. https://doi.org/10.1038/nclimate2837

2. Ashrafi M, Azimi-Moqadam MR, Moradi P, MohseniFard E, Shekari F, Kompany-Zareh M. Effect of drought stress on metabolite adjustments in drought tolerant and sensitive thyme. Plant Physiol Biochem. 2018;132:391-399. https://doi.org/1016/j.plaphy.2018.09.009

3. Cochavi A, Rachmilevitch S, Bel G. The effect of irrigation regimes on plum (Prunus cerasifera) root system development dynamics. Plant Biosyst. 2019;153:529-537.

https://doi.org/10.1080/11263504.2018.1508087

4. Calvo OC, Franzaring J, Schmid I, Müller M, Brohon N, Fangmeier A. Atmospheric $\mathrm{CO}_{2}$ enrichment and drought stress modify root exudation of barley. Global Change Biol. 2016;23: 1292-1304. https://doi.org/10.1111/gcb.13503

5. Hu LF, Robert CAM, Cadot S, Zhang X, Ye M, Li BB, et al. Root exudate metabolites drive plant-soil feedbacks on growth and defense by shaping the rhizosphere microbiota. Nat Commun. 2018;9:2738. https://doi.org/10.1038/s41467-018-05122-7

6. Lata R, Chowdhury S, Gond SK, White JFJr. Induction of abiotic stress tolerance in plants by endophytic microbes. Lett Appl Microbiol. 2018;66:268-276.

https://doi.org/10.1111/lam.12855

7. Fitzpatrick CR, Mustafa Z, Viliunas J. Soil microbes alter plant fitness under competition and drought. J Evol Biol. 2019;32:438-450. https://org/10.1111/jeb.13426

8. He C, Wang W, Hou J. Plant growth and soil microbial impacts of enhancing licorice with inoculating dark septate endophytes under drought stress. Front Microbiol. 2019;10:2277.

https://doi.org/10.3389/fmicb.2019.02277 
9. Saleem M, Law AD, Sahib MR, Pervaiz ZH, Zhang Q. Impact of root system architecture on rhizosphere and root microbiome. Rhizosphere. 2018;6:47-51.

https://doi.org/10.1016/j.rhisph.2018.02.003

10. Hou LF, Yu J, Zhao LL, He XL. Dark septate endophytes improve the growth and the tolerance of Medicago sativa and Ammopiptanthus mongolicus under cadmium stress. Front Microbiol. 2020; 10:3061. https://org/10.3389/fmicb.2019.03061

11. Li X, He XL, Hou LF, Ren Y, Wang SJ, Su F. Dark septate endophytes isolated from a xerophyte plant promote the growth of Ammopiptanthus mongolicus under drought condition. Sci Rep. 2018; 8:7896. https://doi.org/10.1038/s41598-018-26183-0

12. Jumpponen A, Trappe JM. Dark septate endophytes: a review of facultative biotrophic rootcolonizing fungi. New Phytol. 1998;140:295-310.

https://doi.org/10.1046/j.1469-8137.1998.00265.x

13. Bacon CW, White JF. Functions, mechanisms and regulation of endophytic and epiphytic microbial communities of plants. Symbiosis. 2016;68:87-

https://doi.org/10.1007/s13199-015-0350-2

14. Ruotsalainen AL, Eskelinen A. Root fungal symbionts interact with mammalian herbivory, soil nutrient availability and specific habitat conditions. Oecologia. 2011;166:807-817.

https://doi.org/10.1007/s00442-011-1928-x

15. Fiorentino N, Ventorino V, Woo SL, Pepe O, De Rosa A, Gioia L, et al. Trichoderma-based biostimulants modulate rhizosphere microbial populations and improve $\mathrm{N}$ uptake efficiency, yield, and nutritional quality of leafy vegetables. Front Plant Sci. 2018;9:743.

https://doi.org/10.3389/fpls.2018.00743

16. Changey F, Meglouli H, Fontaine J, Magnin-Robert M, Tisserant B, Lerch TZ, et al. Initial microbial status modulates mycorrhizal inoculation effect on rhizosphere microbial communities. Mycorrhiza. 2019;29:475- https://doi.org/10.1007/s00572-019-00914-1

17. Tian L, Shi SH, Ma LN, Zhou X, Luo SS, Zhang JF, et al. The effect of Glomus intraradices on the physiological properties of Panax ginseng and on rhizospheric microbial diversity. J Ginseng Res. 2019;43:77-85. http://dx.doi.org/10.1016/j.jgr.2017.08.005

18. Han LJ, Wang ZY, Li N, Wang YH, Feng JT, Zhang X. Bacillus amyloliquefaciens B1408 suppresses Fusarium wilt in cucumber by regulating the rhizosphere microbial community. Appl Soil Ecol. 2019;136:55-66. https://doi.org/10.1016/j.apsoil.2018.12.011 
19. Mayerhofer MS, Kernaghan G, Harper KA. The effects of fungal root endophytes on plant growth: a meta-analysis. Mycorrhiza. 2013;23:119-128.

https://doi.org/10.1007/s00572-012-0456-9

20. Furtado BU, Szymańska S, Hrynkiewicz K. A window into fungal endophytism in Salicornia

europaea: deciphering fungal characteristics as plant growth promoting agents. Plant Soil. 2019;445:577-594. https://doi.org/10.1007/s11104-019-04315-3

21. Rizzato G, Scalabrin E, Radaelli M, Capodaglio G, Piccolo O. A new exploration of licorice metabolome. Food Chem. 2017;221:959-968. https://doi.org/10.1016/j.foodchem.2016.11.068

22. Zhang WJ, Xie ZC, Zhang XJ, Lang DY, Zhang XH. Growth-promoting bacteria alleviates drought stress of Glycyrrhiza uralensis through improving photosynthesis characteristics and water status. J Plant Interact. 2019;14:580-589. https://doi.org/10.1080/17429145.2019.1680752

23. Li L, Sinkko H, Montonen L, Wei GH, Lindstrom K, Rasanen LA. Biogeography of symbiotic and other endophytic bacteria isolated from medicinal Glycyrrhiza species in China. FEMS Microbiol Ecol. 2012;79:46-68. https://doi.org/10.1111/j.1574-6941.2011.01198.x

24. Egamberdieva D, Li L, Lindstrom K, Rasanen LA. A synergistic interaction between salt- tolerant Pseudomonas and Mesorhizobium strains improves growth and symbiotic performance of liquorice (Glycyrrhiza uralensis) under salt stress. Appl Microbiol Biotechnol. 2016;100:2829-2841. https://doi.org/10.1007/s00253-015-7147-3

25. Hartman K, van der Heijden MGA, Roussely-Provent V, Walser JC, Schlaeppi J. Deciphering composition and function of the root microbiome of a legume plant. Microbiome. 2017;5:2.

https://doi.org/10.1186/s40168-016-0220-z

26. Zhang YZ, Xu J, Riera N, Jin T, Li JY, Wang NA. Huanglongbing impairs the rhizosphere- torhizoplane enrichment process of the citrus root-associated microbiome. Microbiome. 2017;5:97. https://doi.org/10.1186/s40168-017-0304-4

27. Young E, Carey M, Meharg AA, Meharg C. Microbiome and ecotypic adaption of Holcus lanatus (L.) to extremes of its soil pH range, investigated through transcriptome sequencing. Microbiome. 2018;6:48. https://doi.org/10.1186/s40168-018-0434-3

28. Suleiman MK, Dixon K, Commander L, Nevill P, Quoreshi AM, Bhat NR, et al. Assessment of the diversity of fungal community composition associated with Vachellia pachyceras and its rhizosphere soil from Kuwait Desert. Front Microbiol. 2019;10:63.

https://doi.org/10.3389/fmicb.2019.00063

29. Dai LX, Zhang GC, Yu ZP, Ding H, Xu Y, Zhang ZM. Effect of drought stress and developmental stages on microbial community structure and diversity in peanut rhizosphere soil. Int J Mol Sci. 2019;20:2265. https://doi.org/10.3390/ijms20092265 
30. Lundberg DS, Lebeis SL, Paredes SH, Yourstone S, Gehring J, Malfatti S, et al. Defining the core Arabidopsis thaliana root microbiome. Nature. 2012;488:86-90.

https://doi.org/10.1038/nature11237

31. Bulgarelli D, Garrido-Oter R, Munch PC, Weiman A, Droge J, Pan Y, et al. Structure and function of the bacterial root microbiota in wild and domesticated barley. Cell Host Microbe. 2015;17:392-403. https://doi.org/10.1016/j.chom.2015.01.011

32. Achouak W, Abrouk D, Guyonnet J, Barakat M, Ortet P, Simon L, et al. Plant hosts control microbial denitrification activity. FEMS Microbiol Ecol. 2019;95:fiz021.

https://doi.org/10.1093/femsec/fiz021

33. Ravanbakhsh M, Sasidharan R, Voesenek LACJ, Kowalchuk GA, Jousset A. Microbial modulation of plant ethylene signaling: ecological and evolutionary consequences. Microbiome. 2018;6:52. https://doi.org/10.1186/s40168-018-0436-1

34. Ullah A, Akbar A, Luo Q, Khan AH, Manghwar H, Shaban M, et al. Microbiome diversity in cotton rhizosphere under normal and drought conditions. Microb Ecol. 2019;77:429-439.

https://doi.org/10.1007/s00248-018-1260-7

35. Raklami A, Bechtaoui N, Tahiri A, Anli M, Meddich A, Oufdou K. Use of rhizobacteria and mycorrhizae consortium in the open field as a strategy for improving crop nutrition, productivity and soil fertility. Front Microbiol. 2019;10:1106. https://doi.org/13389/fmicb.2019.01106

36. Zhang F, Xu X, Huo Y, Xiao Y. Trichoderma-inoculation and mowing synergistically altered soil available nutrients, rhizosphere chemical compounds and soil microbial community, potentially driving alfalfa growth. Front Microbiol. 2019;9:3241. https://doi.org/10.3389/fmicb.2018.03241

37. Gonçalves VN, Cantrell CL, Wedge DE, Ferreira MC, Soares MA, Jacob MR, et al. Fungi associated with rocks of the Atacama Desert: Taxonomy, distribution, diversity, ecology and bioprospection for bioactive compounds. Environ Microbiol. 2016;18:232-245.

https://doi.org/10.1111/1462-2920.13005

38. Surono, Narisawa K. The inhibitory role of dark septate endophytic fungus Phialocephala fortinii against Fusarium disease on the Asparagus officinalis growth in organic source conditions. Biol Control. 2018;121:159-167. https://doi.org/10.1016/j.biocontrol.2018.02.017

39. Vergara C, Araujo KEC, Alves LS, Souza SRD, Santos LA, Santa-Catarina C, et al. Contribution of dark septate fungi to the nutrient uptake and growth of rice plants. Brazilian J Microbiol. 2018;49:67-78. https://doi.org/10.1016/j.bjm.2017.04.010

40. Lin YB, Yea YM, Hu YM, Shi HK. The variation in microbial community structure under different heavy metal contamination levels in paddy soils. Ecotox Environ Safe. 2019;180:557- 564. https://doi.org/10.1016/j.ecoenv.2019.05.057 
41. Khan AL, Asaf S, Abed KMM, Chai YN, Al-Rawahi AN, Mohanta TK, et al. Rhizosphere microbiome of arid land medicinal plants and extra cellular enzymes contribute to their abundance. Microorganisms. 2020, 8:213. https://doi.org/10.3390/microorganisms8020213

42. Khan I, Aftab M, Shakir S, Ali M, Qayyum S, Rehman MU, et al. Mycoremediation of heavy metal (Cd and $\mathrm{Cr}$ )-polluted soil through indigenous metallotolerant fungal isolates. Environ Monit Assess. 2019;191:585. https://doi.org/10.1007/s10661-019-7769-5

43. Sanguin H, Sarniguet A, Gazengel K, Moenne-Loccoz Y, Grundmann GL. Rhizosphere bacterial communities associated with disease suppressiveness stages of take-all decline in wheat monoculture. New Phytol. 2010;184:694-707. https://doi.org/10.1111/j.1469-8137.2009.03010.x

44. Singh H. Desiccation and radiation stress tolerance in cyanobacteria. J Basic Microbiol. 2018; 58:813-826. https://doi.org/10.1002/jobm.201800216

45. Shen ZZ, Ruan YZ, Chao X, Zhang J, Li R, Shen QR. Rhizosphere microbial community manipulated by 2 years of consecutive biofertilizer application associated with banana Fusarium wilt disease suppression. Biol Fert Soils. 2015;51:1-10.

https://doi.org/10.1007/s00374-015-1002-7

46. Wu ZH, Liu QS, Li ZY, Cheng W, Sun JM, Guo ZH, et al. Environmental factors shaping the diversity of bacterial communites that promote rice production. BMC Microbiol. 2018;18:51.

https://doi.org/10.1186/s12866-018-1174-z

47. Kisa M, Sanon A, Thioulouse J, Assigbetse K, Sylla S, Spichiger R, et al. Arbuscular mycorrhizal symbiosis can counterbalance the negative influence of the exotic tree species Eucalyptus camaldulensis on the structure and functioning of soil microbial communities in a sahelian soil. FEMS Microbiol Ecol. 2007;62:32-44. https://doi.org/10.1111/j.1574-6941.2007.00363.x

48. Lackner M, de Hoog GS, Yang L, Moreno LF, Ahmed SA, Andreas F, et al. Proposed nomenclature for Pseudallescheria, Scedosporium and related genera. Fungal Divers. 2014;67: 1-10. https://doi.org/10.1007/s13225-014-0295-4

49. Sandoval-Denis M, Gené J, Sutton DA, Cano-Lira JF, de Hoog GS, Decock CA, et al. Redefining Microascus, Scopulariopsis and allied genera. Persoonia. 2016;36:1-36.

https://doi.org/10.3767/003158516X688027

50. Contreras-Cornejo HA, Macías-Rodríguez L, del-Val E, Larsen J. Ecological functions of Trichoderma and their secondary metabolites in the rhizosphere: interactions with plants. FEMS Microbiol Ecol. 2016;92: fiw036. https://doi.org/10.1093/femsec/fiw036

51. Fountain JC, Koh J, Yang LM, Pandey MK, Nayak SN, Bajaj P, et al. Proteome analysis of Aspergillus favus isolate-specific responses to oxidative stress in relationship to afatoxin production capability. Sci Rep. 2018;8:3430. https://doi.org/10.1038/s41598-018-21653-x 
52. Chatterjee S, Sharma S, Prasad RK, Datta S, Dubey D, Meghvansi MK, et al. Cellulase enzyme based biodegradation of cellulosic materials: An overview. South Asian J Exp Biol. 2015;5: 271-

53. Rollin-Pinheiro R, de Meirelles JV, Vila TVM, Fonseca BB, Alves V, Frases S, et al. Biofilm formation by Pseudallescheria / Scedosporium species: A comparative study. Front Microbiol. 2017;8:1568. https://doi.org/10.3389/fmicb.2017.01568

54. Igiehon NO, Babalola OO, Aremu BR. Genomic insights into plant growth promoting rhizobia capable of enhancing soybean germination under drought stress. BMC Microbiol. 2019;19:159. https://doi.org/10.1186/s12866-019-1536-1

55. Khan N, Bano A, Babar MA. Metabolic and physiological changes induced by plant growth regulators and plant growth promoting rhizobacteria and their impact on drought tolerance in Cicer arietinum. PLoS ONE 2019;14:e0213040. https://doi.org/10.1371/journal.pone.0213040

56. Wang L, Lin H, Dong YB, Li B, He YH. Effects of endophytes inoculation on rhizosphere and endosphere microecology of Indian mustard (Brassica juncea) grown in vanadium- contaminated soil and its enhancement on phytoremediation. Chemosphere. 2020;240:124891. https://doi.org/10.1016/j.chemosphere. 2019.124891

57. Wang J, Cao JM, Chen DX, Qiu J, Wang XQ, Feng C, et al. Antimicrobial effect and components analysis of volatile organic compounds from Bacillus pumilus Sci Agric Sin. 2018;51: 1908-1919.

58. Wang XR, Peng ZQ, Sun XL, Liu DB, Chen S, Li F, Xia HM, et al. The FPase properties and morphology changes of a cellulolytic bacterium, Sporocytophaga JL-01, on decomposing filter paper cellulose. J Gen Appl Microbiol. 2012;58:429-436. https://doi.org/10.2323/jgam.58.429

59. Zhang H, Sun H, Yang R, Li S, Zhou M, Gao T, et al. Complete genome sequence of a psychotrophic Pseudarthrobacter sulfonivorans strain Ar51 (CGMCC 4.7316), a novel crude oil and multi benzene compounds degradation strain. J Biotechnol. 2016;231:81-

https://doi.org/10.1016/j.jbiotec.2016.04.010

60. Rodríguez-Caballero G, Caravaca F, Fernández-González AJ, Alguacil MM, Fernández-López M, Roldán A. Arbuscular mycorrhizal fungi inoculation mediated changes in rhizosphere bacterial community structure while promoting revegetation in a semiarid ecosystem. Sci Total Environ. 2017;584-585:838-848. https://doi.org/10.1016/j.scitotenv.2017.01.128

61. Rowell DL. Soil Science: Methods and Applications. London: Longman Group. 1994.

62. Olsen SR, Cole CV, Watanabe FS, Dean LA. Estimation of Available Phosphorus in Soils by Extraction with Sodium Bicarbonate. USDA Circular 939. Washington, DC: US Department of Agriculture, 1-18. 1954.

63. Jackson ML. Soil Chemical Analysis. New Delhi: Prentice Hall of India Pvt. Ltd, 38-56. 1973.

64. Xu N, Tan G, Wang H, Gai XP. Effect of biochar additions to soil on nitrogen leaching, microbial biomass and bacterial community structure. Eur J Soil Biol. 2016;74:1-8. 
65. Adams RI, Miletto M, Taylor JW, Bruns TD. Dispersal in microbes: fungi in indoor air are dominated by outdoor air and show dispersal limitation at short distances. ISME J. 2013;7: 1262-1273. https://doi.org/10.1038/ismej.2013.28

66. Magoč T, Salzberg SL. FLASH: fast length adjustment of short reads to improve genome assemblies. Bioinformatics. 2011;27:2957-2963. https://doi.org/10.1093/bioinformatics/btr507

67. Chen $\mathrm{H}$, Boutros PC. VennDiagram: A package for the generation of highly- customizable Venn and Euler diagrams in R. BMC Bioinform. 2011;12:35.

https://doi.org/10.1186/1471-2105-12-35

68. Maughan H, Wang PW, Diaz Caballero J, Fung P, Gong Y, Donaldson SL, et al. Analysis of the cystic fibrosis lung microbiota via serial Illumina sequencing of bacterial 16S rRNA hypervariable regions. PLoS ONE. 2012;7:e45791. https://doi.org/10.1371/journal.pone.0045791

69. Chen B, Teh BS, Sun C, Hu S, Lu X, Boland W, et al. Biodiversity and activity of the gut microbiota across the life history of the insect herbivore Spodoptera littoralis. Sci Rep. 2016;6: 29505. https://doi.org/10.1038/srep29505

70. Oksanen J, Blanchet FG, Friendly M, Kindt R, Legendre P, McGlinn D, et al. Vegan: community ecology package. R package version 2.4-1. 2016.

71. Yao H, Sun X, He C, Maitra P, Li XC, Guo LD. Phyllosphere epiphytic and endophytic fungal community and network structures differ in a tropical mangrove ecosystem. Microbiome. 2019;7:57. https://doi.org/10.1186/s40168-019-0671-0

72. Huang Y, Xiao X, Huang HY, Jing JQ, Zhao HJ, Wang L, et al. Contrasting beneficial and pathogenic microbial communities across consecutive cropping fields of greenhouse strawberry. Appl Microbiol Biot. 2018;102:5717-5729. https://doi.org/10.1007/s00253-018-9013-6

73. Shannon P, Markiel A, Ozier O, Baliga NS, Wang JT, Ramage D, et al. Cytoscape: a software environment for integrated models of biomolecular interaction networks. Genome Res. 2003;13: 2498- https://doi.org/10.1101/gr.1239303

74. Nguyen $\mathrm{N} \mathrm{H}$, Song Z W, Bates S T, et al. FUNguild: An open annotation tool for parsing fungal community datasets by ecological guild. Fungal Ecol. 2015;20:241https://doi.org/10.1016/j.funeco.2015.06.006

75. Mendes LW, Raaijmakers JM, de Hollander M, Mendes R, Tsai SM. Influence of resistance breeding in common bean on rhizosphere microbiome composition and function. ISME J. 2018; 12:212-224. https://doi.org/10.1038/ismej.2017.158

\section{Tables}

Table 1 Diversity and richness index of licorice rhizosphere soil fungi 


\begin{tabular}{|lllll|}
\hline Samples & Shannon & Simpson & Ace & Chao1 \\
\hline DCK & $1.63 \pm 0.41 \mathrm{c}$ & $0.35 \pm 0.08 \mathrm{a}$ & $324.22 \pm 26.51 \mathrm{~d}$ & $285.46 \pm 38.25 \mathrm{e}$ \\
\hline DPP & $3.62 \pm 0.48 \mathrm{a}$ & $0.07 \pm 0.03 \mathrm{~d}$ & $721.28 \pm 59.33 \mathrm{a}$ & $675.46 \pm 75.47 \mathrm{a}$ \\
\hline DAV & $1.63 \pm 0.15 \mathrm{c}$ & $0.37 \pm 0.06 \mathrm{a}$ & $430.36 \pm 43.16 \mathrm{c}$ & $352.79 \pm 39.38 \mathrm{~d}$ \\
\hline NCK & $2.81 \pm 0.27 \mathrm{~b}$ & $0.16 \pm 0.04 \mathrm{c}$ & $590.11 \pm 67.24 \mathrm{~b}$ & $536.27 \pm 40.08 \mathrm{~b}$ \\
\hline NPP & $2.32 \pm 0.43 \mathrm{bc}$ & $0.28 \pm 0.02 \mathrm{~b}$ & $558.51 \pm 33.29 \mathrm{~b}$ & $522.83 \pm 39.48 \mathrm{~b}$ \\
\hline NAV & $2.08 \pm 0.31 \mathrm{bc}$ & $0.31 \pm 0.06 \mathrm{ab}$ & $494.84 \pm 42.57 \mathrm{bc}$ & $435.04 \pm 37.39 \mathrm{c}$ \\
\hline
\end{tabular}

Note: Different letters in the same column indicate significant differences between different samples $(P<$ 0.05). NCK, non-inoculation under well-watered condition; NPP, inoculation with Paraboeremia putaminum under well-watered condition; NAV, inoculation with Acrocalymma vagum under well-watered condition; DCK, non-inoculation under drought stress; DPP, inoculation with Paraboeremia putaminum under drought stress; DAV, inoculation with Acrocalymma vagum under drought stress.

Table 2 Diversity and richness index of licorice rhizosphere soil bacteria

\begin{tabular}{|lllll|}
\hline Samples & Shannon & Simpson & Ace & Chao 1 \\
\hline DCK & $6.45 \pm 0.29 \mathrm{ab}$ & $0.0067 \pm 0.003 \mathrm{c}$ & $4150.99 \pm 429.98 \mathrm{~b}$ & $4142.55 \pm 480.04 \mathrm{bc}$ \\
\hline DPP & $6.78 \pm 0.05 \mathrm{a}$ & $0.0045 \pm 0.001 \mathrm{c}$ & $5203.69 \pm 651.62 \mathrm{a}$ & $4906.27 \pm 211.29 \mathrm{a}$ \\
\hline DAV & $6.34 \pm 0.31 \mathrm{ab}$ & $0.0066 \pm 0.002 \mathrm{c}$ & $4289.02 \pm 341.35 \mathrm{~b}$ & $4068.12 \pm 477.16 \mathrm{bc}$ \\
\hline NCK & $5.78 \pm 0.13 \mathrm{~b}$ & $0.0251 \pm 0.008 \mathrm{a}$ & $4404.73 \pm 226.99 \mathrm{~b}$ & $3795.56 \pm 358.63 \mathrm{c}$ \\
\hline NPP & $6.43 \pm 0.21 \mathrm{ab}$ & $0.0102 \pm 0.002 \mathrm{~b}$ & $5009.08 \pm 441.12 \mathrm{ab}$ & $4485.35 \pm 85.86 \mathrm{~b}$ \\
\hline NAV & $6.54 \pm 0.25 \mathrm{ab}$ & $0.0055 \pm 0.002 \mathrm{c}$ & $5259.61 \pm 630.63 \mathrm{a}$ & $4322.72 \pm 508.26 \mathrm{~b}$ \\
\hline
\end{tabular}

Note: Different letters in the same column indicate significant differences between different samples $(P<$ 0.05). NCK, non-inoculation under well-watered condition; NPP, inoculation with Paraboeremia putaminum under well-watered condition; NAV, inoculation with Acrocalymma vagum under well-watered condition; DCK, non-inoculation under drought stress; DPP, inoculation with Paraboeremia putaminum under drought stress; DAV, inoculation with Acrocalymma vagum under drought stress.

\section{Figures}


A

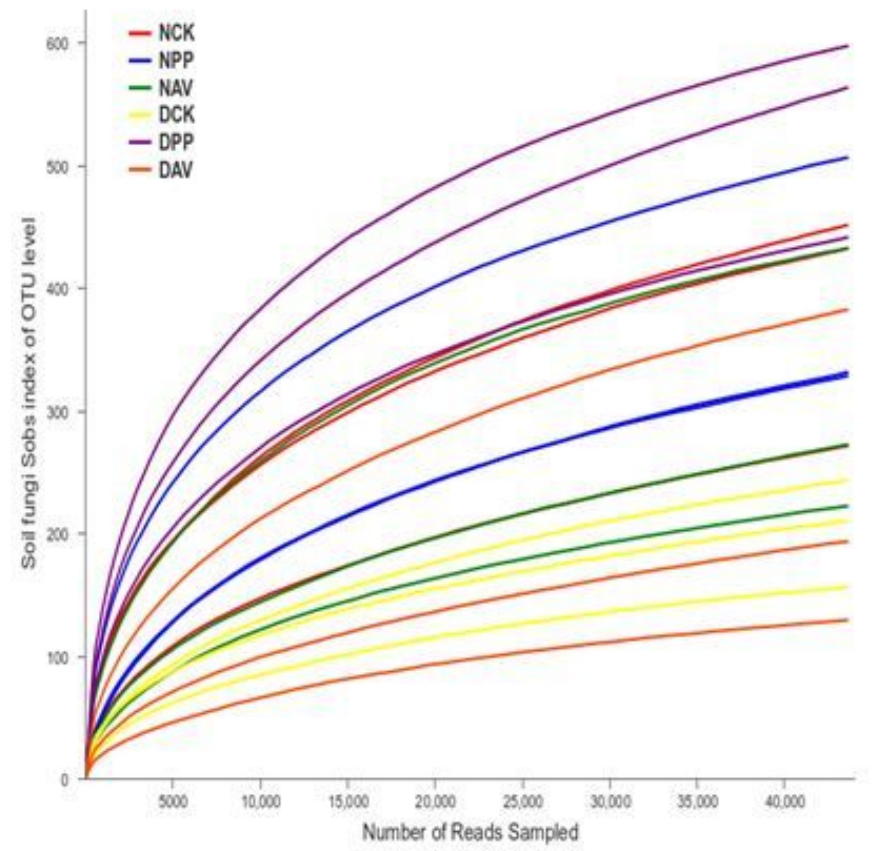

B

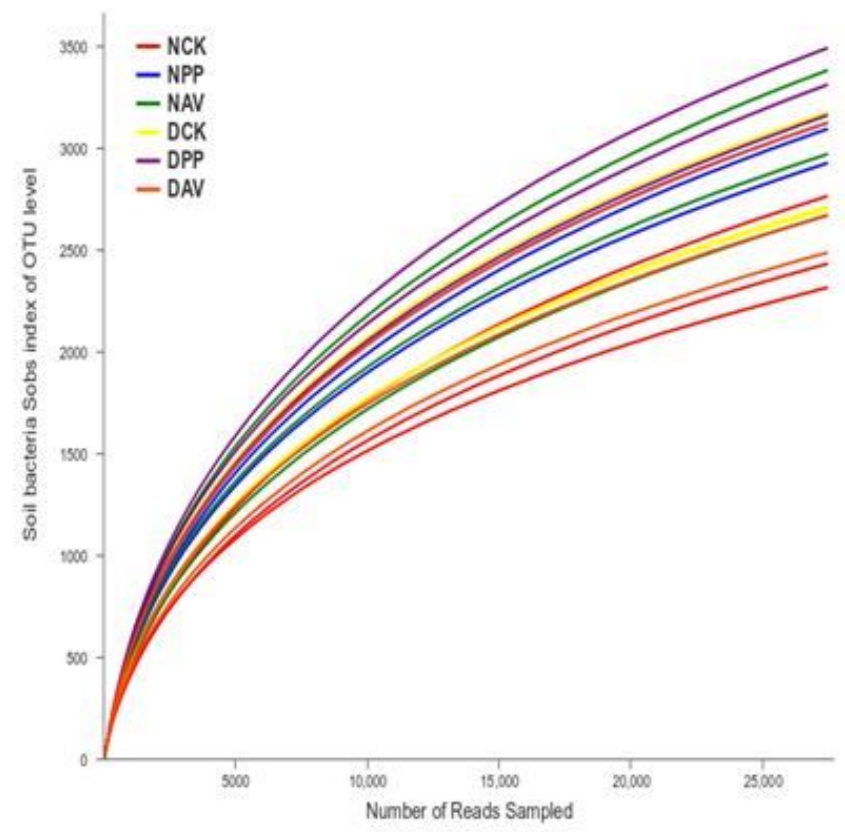

Figure 1

Rarefaction curves for the fungal (A) and bacterial (B) operational taxonomic units (OTUs) in licorice rhizosphere. NCK, non-inoculation under well-watered condition; NPP, inoculation with Paraboeremia putaminum under well-watered condition; NAV, inoculation with Acrocalymma vagum under well-watered condition; DCK, non-inoculation under drought stress; DPP, inoculation with Paraboeremia putaminum under drought stress; DAV, inoculation with Acrocalymma vagum under drought stress.

A

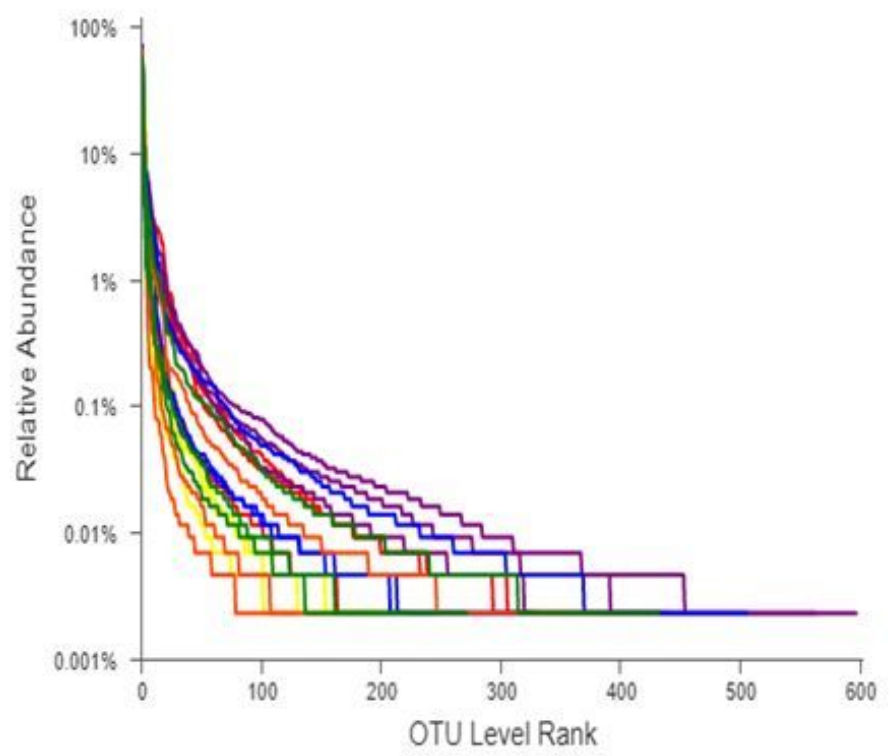

B

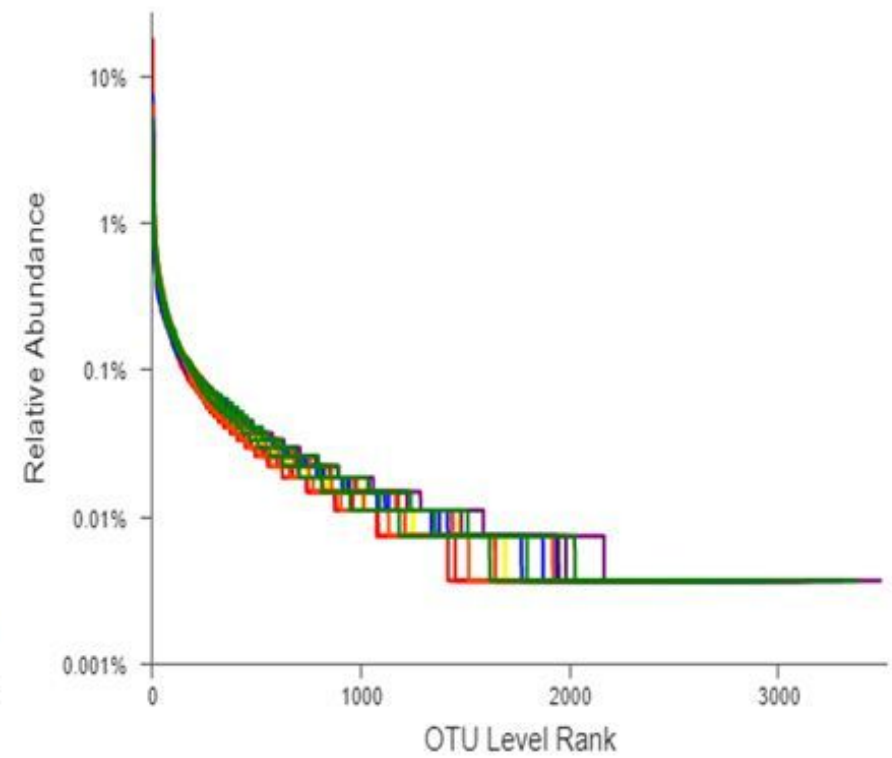


Figure 2

Ranking by the abundance of the fungal (A) and bacterial (B) operational taxonomic units (OTUs) in licorice rhizosphere.

A
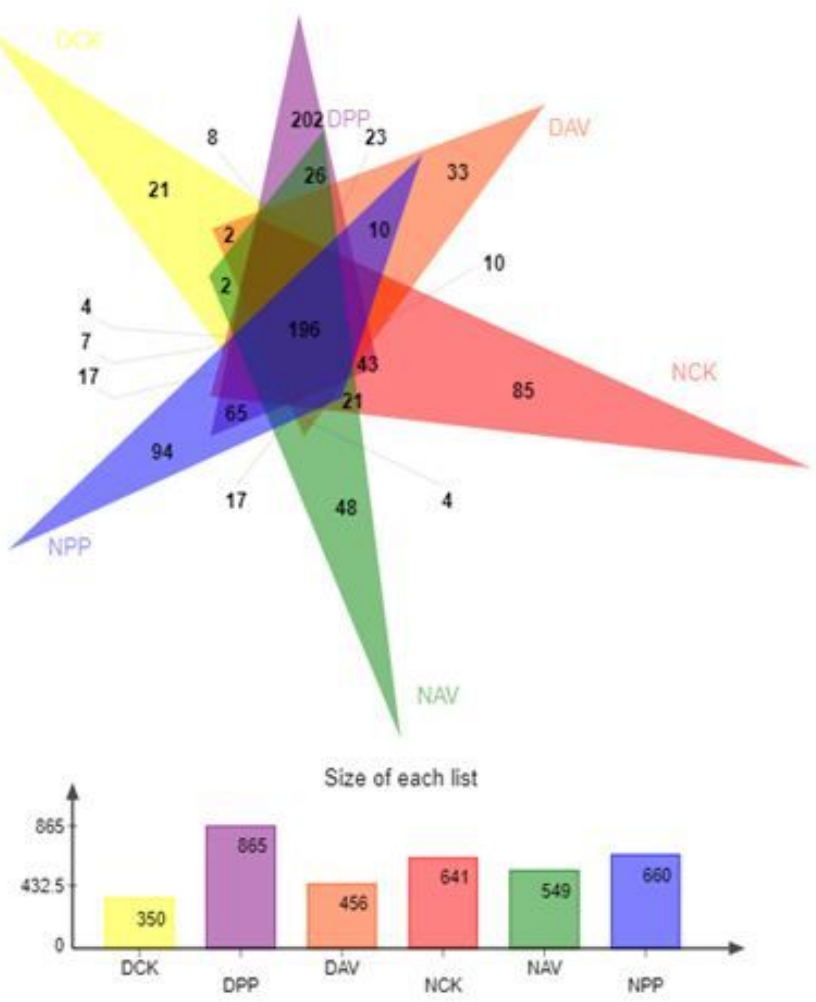

Number of elements: specific (1) or shared by $2,3, \ldots$ lists

\begin{tabular}{|c|c|c|c|c|c|}
\hline 196 & 106 & 112 & 122 & 259 & 483 \\
\hline 6 & 5 & 4 & 3 & 2 & 1
\end{tabular}

B
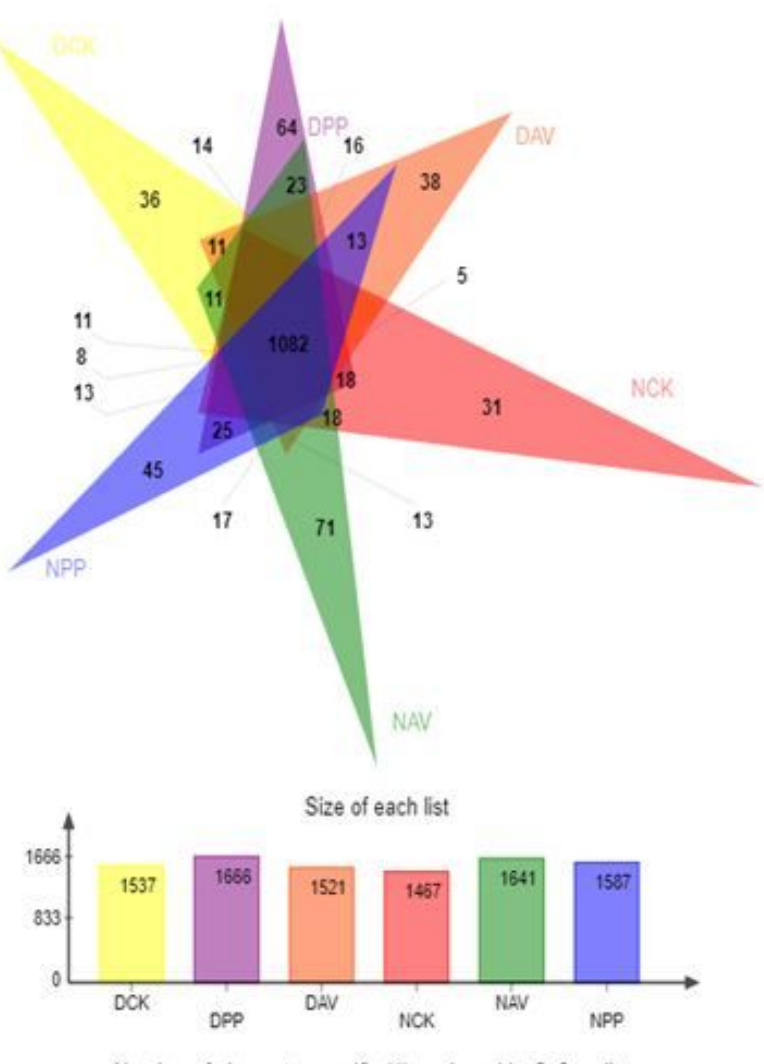

Number of elements: specific (1) or shared by $2,3, \ldots$ lists

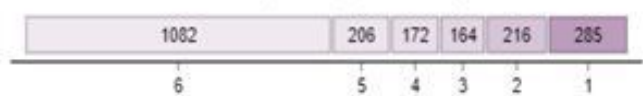

\section{Figure 3}

Venn diagram showing differences in composition of fungal (A) and bacterial (B) OTUs inoculated with different DSE species under well-watered and drought stress, respectively. Each part in the Venn diagram represents one (group) treatment, and the number of circles and circles overlapped represents the number of OTUs shared between the samples (group), while the number without overlapped represents the number of OTUs unique to the samples (group). NCK, non-inoculation under well-watered condition; NPP, inoculation with Paraboeremia putaminum under well-watered condition; NAV, inoculation with Acrocalymma vagum under well-watered condition; DCK, non-inoculation under drought stress; DPP, inoculation with Paraboeremia putaminum under drought stress; DAV, inoculation with Acrocalymma vagum under drought stress. 
A

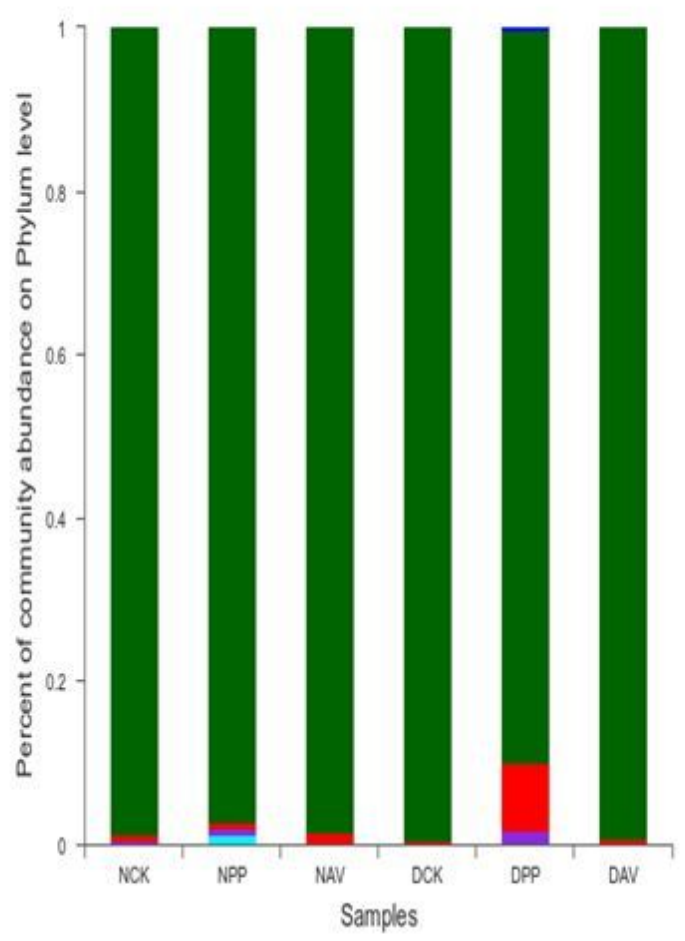

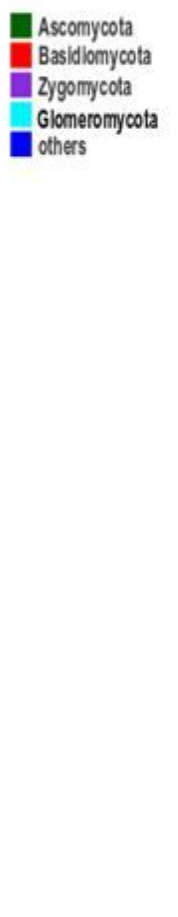

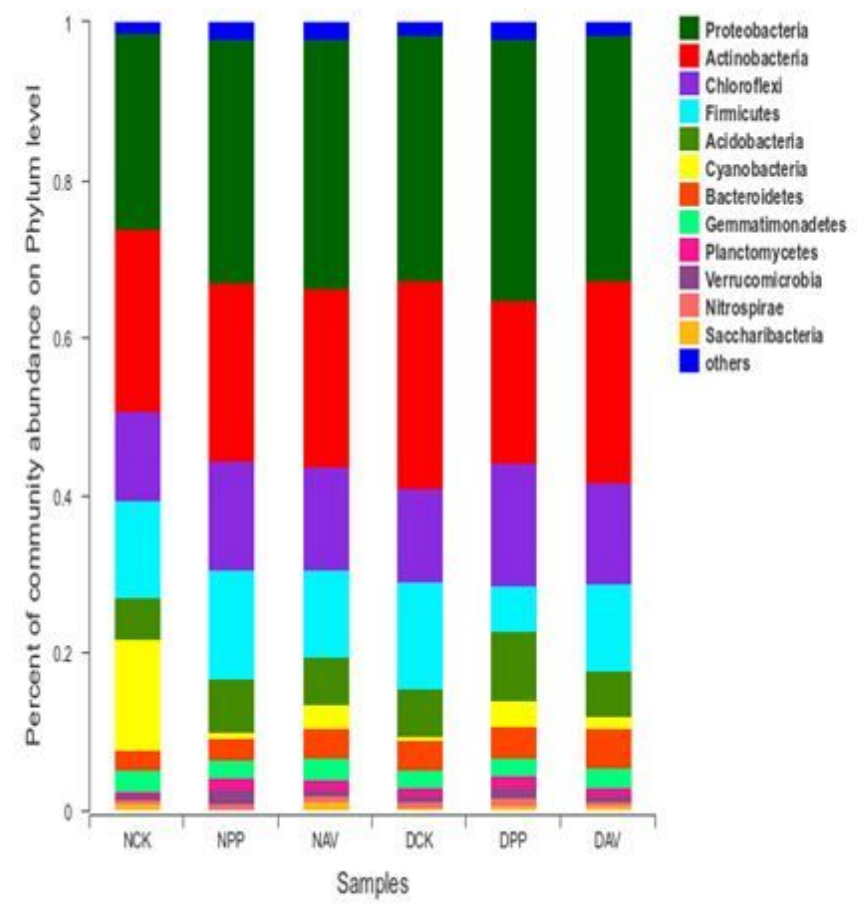

Figure 4

Relative abundance of fungi (A) and bacteria (B) at the phyla level in licorice rhizosphere. NCK, noninoculation under well-watered condition; NPP, inoculation with Paraboeremia putaminum under wellwatered condition; NAV, inoculation with Acrocalymma vagum under well-watered condition; DCK, noninoculation under drought stress; DPP, inoculation with Paraboeremia putaminum under drought stress; $\mathrm{DAV}$, inoculation with Acrocalymma vagum under drought stress. 
A

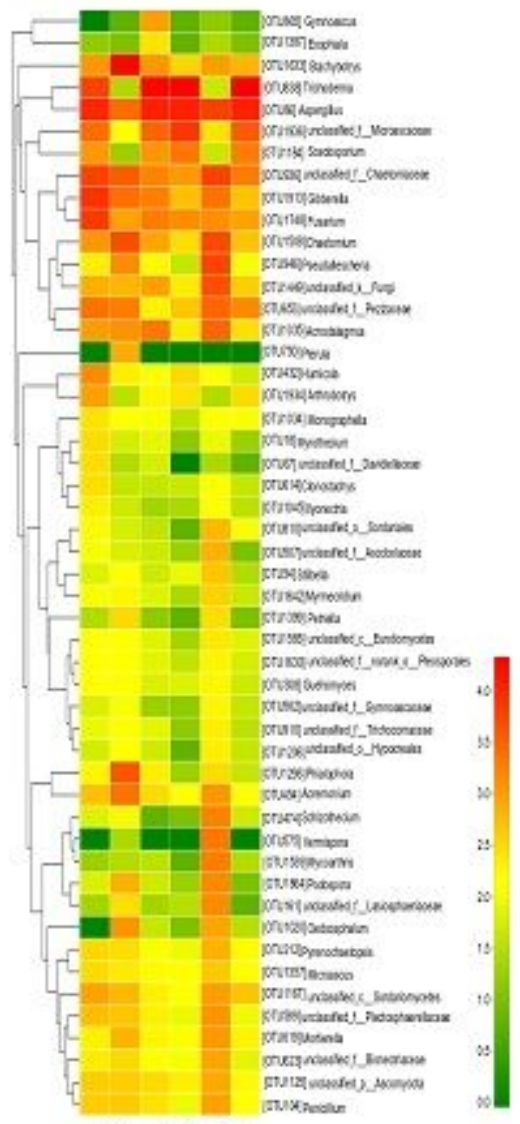

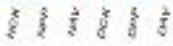

B

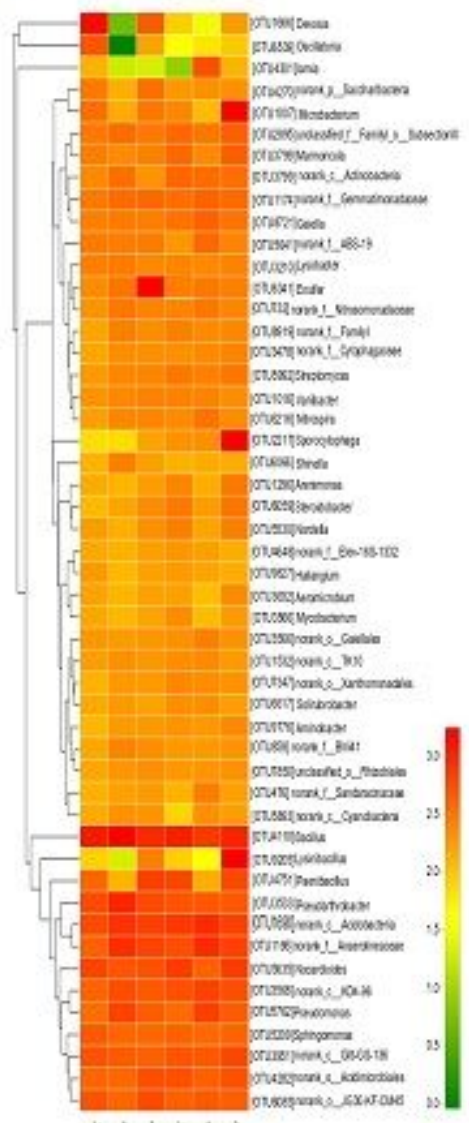

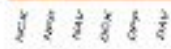

\section{Figure 5}

Heatmap depicting the distribution of relatively abundant fungal (A) and bacterial (B) operational taxonomic units (OTUs) in licorice rhizosphere. The color of each heat map cell indicates the relative abundance of the corresponding fungal and bacterial OTUs. Cluster analysis was performed based on Bray-Curtis similarities. The horizontal represents the treatment information, the vertical represents the clustering situation of different samples, the right represents the species and OTUs annotation, and the left represents the species clustering tree. NCK, non-inoculation under well-watered condition; NPP, inoculation with Paraboeremia putaminum under well-watered condition; NAV, inoculation with Acrocalymma vagum under well-watered condition; DCK, non-inoculation under drought stress; DPP, inoculation with Paraboeremia putaminum under drought stress; DAV, inoculation with Acrocalymma vagum under drought stress. 
A
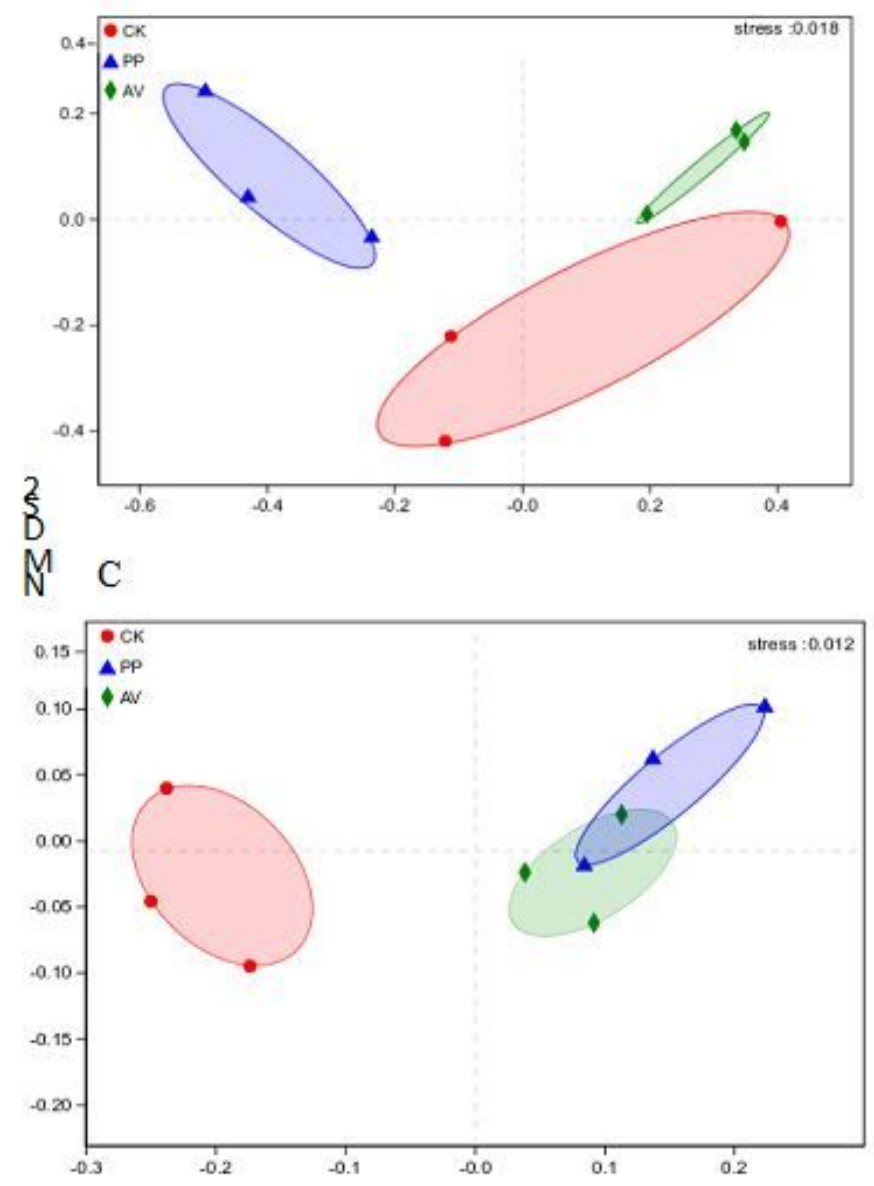

B

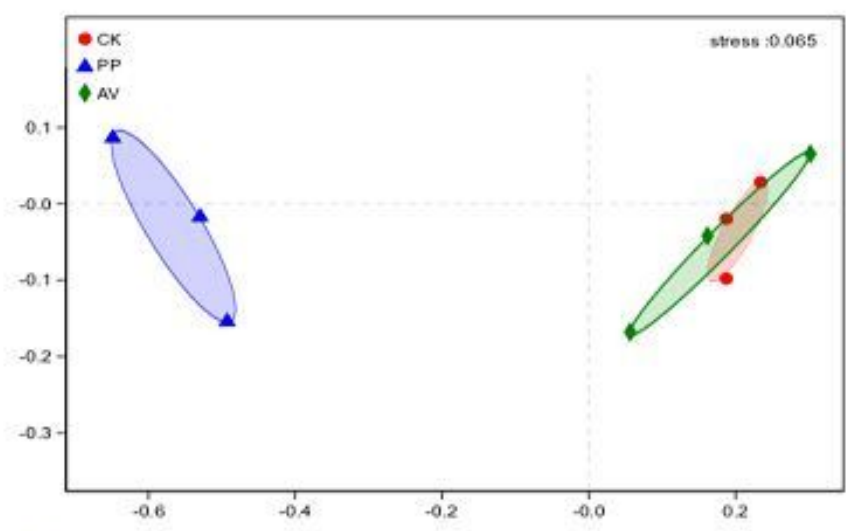

$\mathrm{D}$

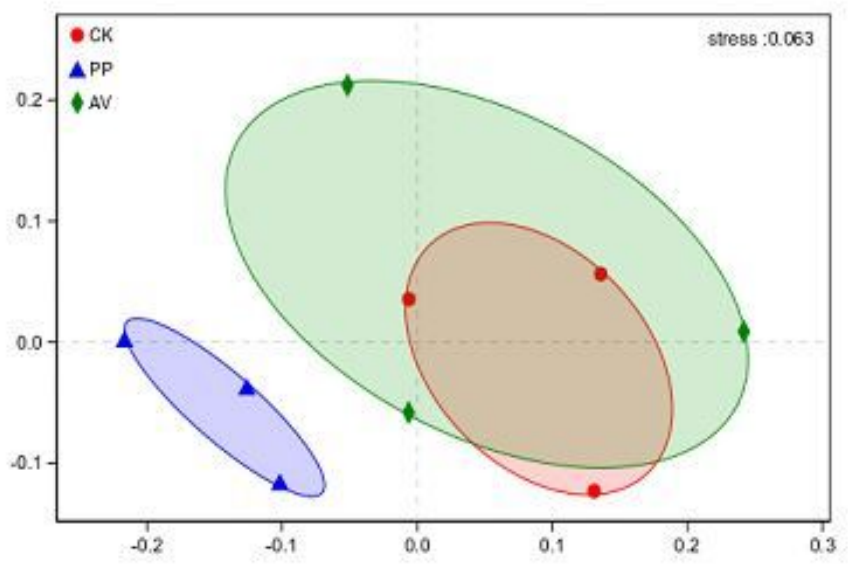

NMDS1

\section{Figure 6}

Non-metric multidimensional scaling (NMDS) ordination of the community composition of fungi and bacteria in licorice rhizosphere under different water regimes. $A$, fungal community under well-watered condition; $\mathrm{B}$, fungal community under drought stress; $\mathrm{C}$, bacterial community under well-watered condition; D, bacterial community under drought stress. CK, non-inoculation; PP, inoculation with Paraboeremia putaminum; $\mathrm{AV}$, inoculation with Acrocalymma vagum. 


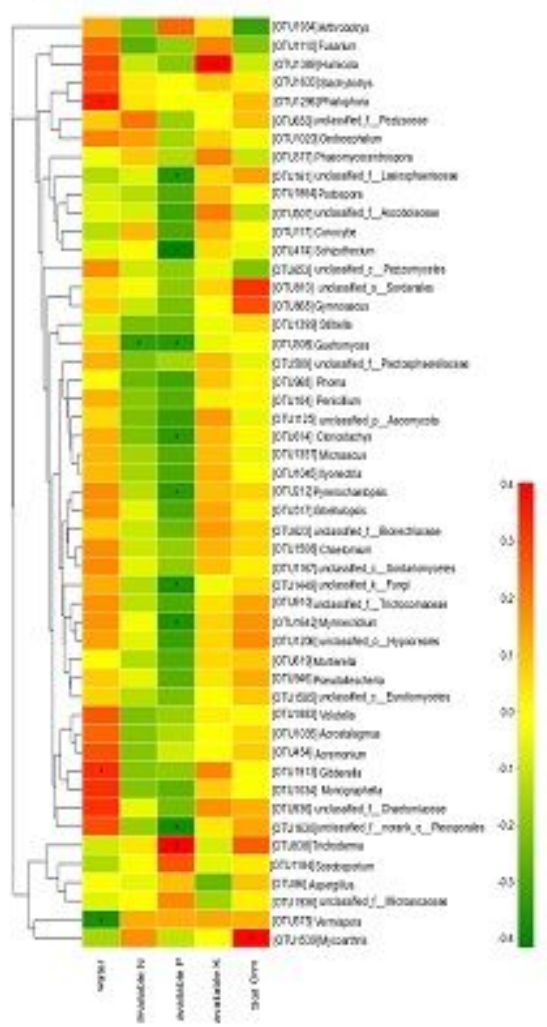

B

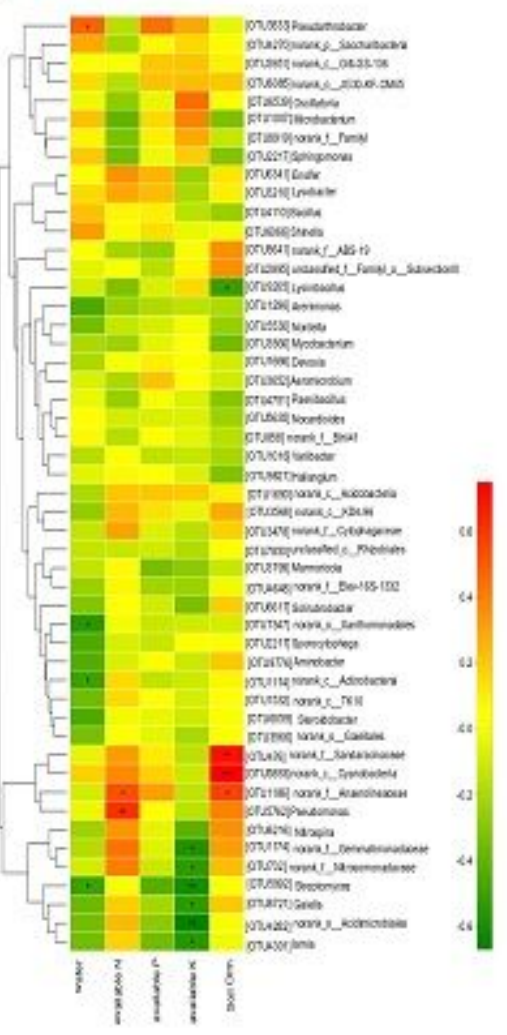

Figure 7

Preferences observed in soil factor-fungus (A) and soil factor-bacterium (B) associations. 


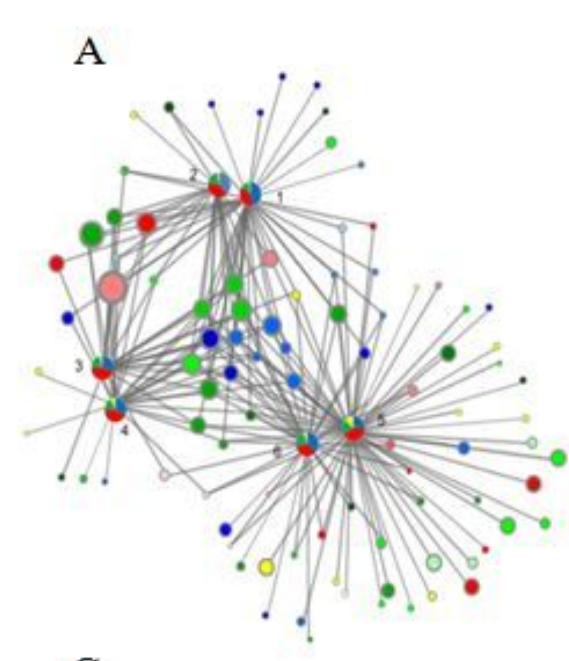

$\mathrm{C}$

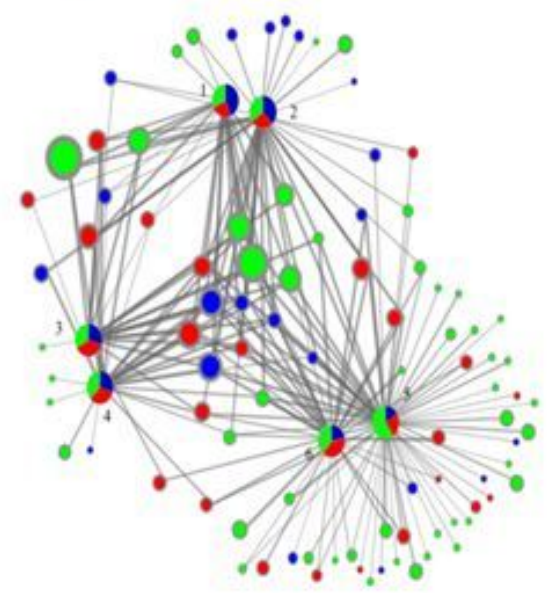

B

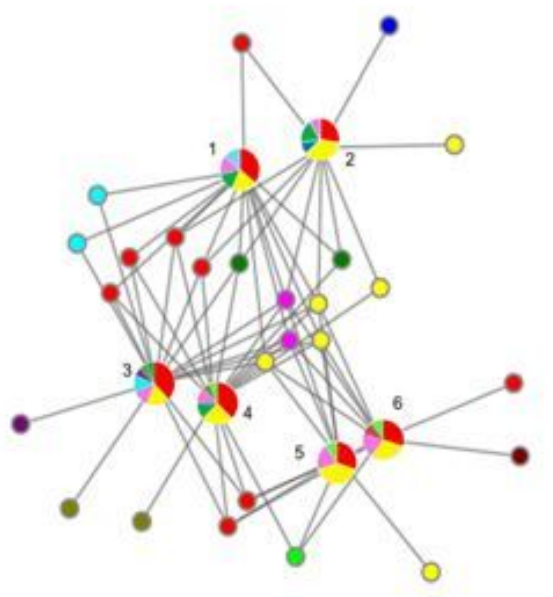

D
I DCK

2 NCK

3 DAV

4 NAV

5 DPP

6 NPP

- Bacillus

- Oscillatoria

- Microbacterium

- Nocardioides

- Pseudarthrobacter

- Sporocytophaga

- Arenimonas

- Ensifer

- Pedomicrobium

Others

Figure 8

1 DCK

2 NCK

3 DAV

4 NAV

5 DPP

6 NPP

Bencficial fungus

Pathogenic fungus

Neutral fungus

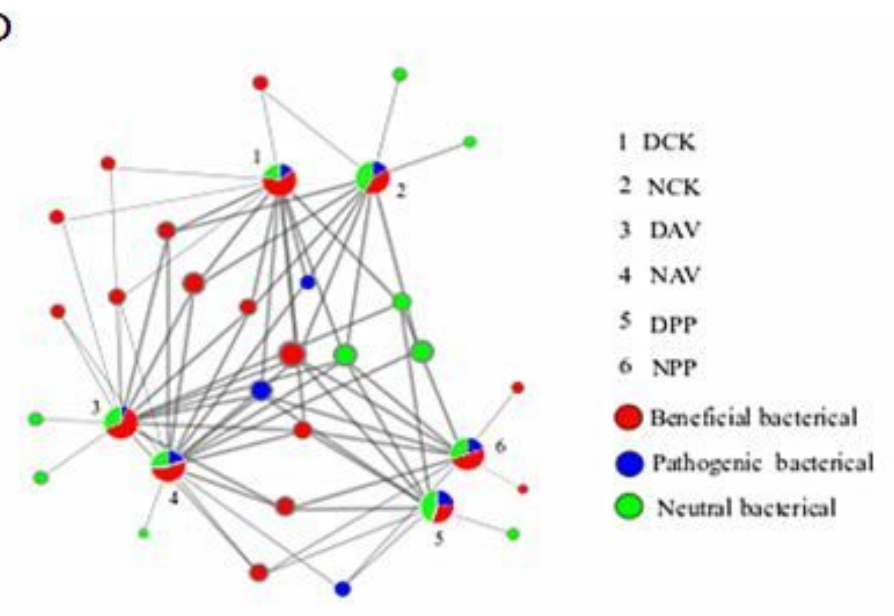

Architecture of the treatment-soil microorganism network. A, visualization of the fungi network at class level; $B$, visualization of the bacterial network at genus level; $C$, visualization network of beneficial, harmful and neutral fungi for licorice plants; $D$, visualization network of beneficial, harmful and neutral bacteria for licorice plants. The size of nodes in A, B, C, D roughly represents the relative abundance of fungal and bacterial operational taxonomic units. 